\title{
Magnetic Pinning of Vortices in a Superconducting Film: The (anti)vortex-magnetic dipole interaction energy in the London approximation
}

\author{
M. V. Milošević, S. V. Yampolskii, \\ Departement Natuurkunde, Universiteit Antwerpen (UIA), \\ Universiteitsplein 1, B-2610 Antwerpen, Belgium
}

(October 24, 2018)

\begin{abstract}
The interaction between a superconducting vortex or antivortex in a superconducting film and a magnetic dipole with in- or out-of-plane magnetization is investigated within the London approximation. The dependence of the interaction energy on the dipole-vortex distance and the film thickness is studied and analytical results are obtained in limiting cases. We show how the short range interaction with the magnetic dipole makes the co-existence of vortices and antivortices possible. Different configurations with vortices and antivortices are investigated.
\end{abstract}

74.60.Ge, 74.76.-w, 74.25.Dw, 74.25.Ha

\section{INTRODUCTION}

The value of the critical current is one of the decisive factors for the usefulness of a superconductor (SC). Large values of the critical currents in superconductors are usually obtained through pinning of vortices to different inhomogeneities in a superconductor. In this respect, external and internal surfaces can be treated as inhomogeneities. Pinning centra are introduced e.g. by locally destroying the crystal structure through e.g. bombardment with high energy particles. Recently, nanostructuring of a superconducting film with a regular area of holest has lead to a large increase of the critical current, in particular at the so-called matching magnetic fields. An alternative approach is to deposit an area of ferromagnetic dots near a superconducting film which acts as very effective trapping centra for the vortices. Recently, it was predicted 3 although not verified, that an increase of the pinning effects by two orders of magnitude can be realized. After substantial progress in the preparation of regular magnetic arrays on superconductors 6 and considering the importance of such structures for magnetic device and storage technologies, these hybrid systems became very interesting both from a theoretical and an experimental point of view. Macroscopic pinning phenomena have already been explored experimentally, 6 but a theoretical analysis of the magnetic and superconducting response in such systems is still lacking. In the majority of recently proposed models 11 the inhomogeneous magnetic field of a ferromagnet induces screening currents in a SC, which, in turn, generate a magnetic field influencing the applied field. Consequently, this problem must be solved self-consistently. Furthermore, the finite thickness of both the superconductor and the ferromagnetic material was not taken into account in previous theoretical treatments.

Other theoretical studies involving finite size ferromagnets were mainly restricted to the problem of a magnetic dot with out-of-plane magnetization embedded in a superconducting film.12. 13 Marmorkos et al.12 solved the non-linear Ginzburg-Landau (GL) equation numerically, with appropriate boundary conditions for an infinitely long ferromagnetic cylinder penetrating the superconducting film, and found a correspondence between the value of the magnetization and the vorticity of the most energetically favorable giant-vortex state. At that time, the interaction between a small ferromagnetic particle, which may be considered as a point magnetic dipole (MD), and a type II superconductor was of interest 14.15 and the magnetostatic energy and levitation force acting on a dipole were calculated, but still within a thin film approximation. In experiments, 16 the magnetic field of the MD was supposed to be weak, and not able to drastically change the structure of the superconducting state in the sample. Obviously, the MD cpuld create additional vortices near the surface, and this process can be described theoretically as proposed in Ref.15, by a simple comparison of the free energies of the system with and without vortex. However, the spontaneous creation of a vortex-antivortex pair as a possible lower energy state was never considered.

Motivated by recent experiments 1 . 18 we focus in this paper on a model system consisting of a type II thin superconducting film (SC) and a magnetic dipole placed above (below) it which acts as a pinning center (Fig. 1). We study in detail, using the London approximation, how the system is perturbed in the neighborhood of the dipole. The superconducting film lies in the $z=0$ xy-plane while the MD is positioned at $(x, y, z)=(0,0, a)$, and is magnetized in the positive z- or x-axis direction. To avoid the proximity effect and exchange of electrons between MD and SC we suppose a thin layer of insulating oxide between them as is usually the case in the experiment.

The paper is organized as follows. In the next section we present the general formalism. In Sec. III, we discuss the pinning potential of the magnetic dipole. In Sec. IV the total interaction energy in the system is calculated in the 
presence of vortex-antivortex pairs, and their most favorable position is determined. The question of stability of such vortex configurations is then analyzed and our conclusions are given in Sec. V.

\section{THEORETICAL FORMALISM}

We consider a magnetic point dipole with magnetization $\vec{M}$, placed outside a type II SC film interacting with a single vortex in the SC. Within the London approximation, the total energy of the stationary magnet-superconductor system is given by 15

$$
\vec{F}=\frac{1}{8 \pi} \int d v_{1}\left[\vec{h}^{2}+\lambda^{2}(\operatorname{rot} \vec{h})^{2}\right]+\frac{1}{8 \pi} \int d v_{2} \vec{h}^{2}
$$

where $\lambda$ is the penetration depth and $\vec{h}$ is the total field present in the system. The integral of the first term is taken over the volume of the SC, and the integral of the second term is taken outside, except for the volume of the dipole. Therefore, we may rewrite Eq. (11) as

$$
\begin{aligned}
F & =\frac{1}{8 \pi} \int d V^{(i)}\left[\vec{h}^{2}+\lambda^{2}(\operatorname{rot} \vec{h})^{2}\right]+\frac{1}{8 \pi} \int d V^{(o)} \vec{h}^{2}-\frac{1}{8 \pi} \int d V^{(m d)} \vec{h}^{2} \\
& =\frac{1}{8 \pi} \int d V\left[\operatorname{rot} \vec{h} \cdot\left(\vec{A}+\lambda^{2} \operatorname{rot} \vec{h}\right)\right]-\frac{1}{8 \pi} \int d \vec{S} \cdot(\vec{h} \times \vec{A})-\frac{1}{8 \pi} \int d V^{(m d)} \vec{h}^{2},
\end{aligned}
$$

where we integrate over the volume inside $V^{(i)}$ and outside $V^{(o)}$ the superconductor, while $V^{(m d)}$ denotes the volume of the magnetic dipole. We choose the surface $S$ far away from the superconductor where we can apply the boundary condition $\vec{h} \rightarrow 0$. Due to the London equation, the field of the magnetic dipole satisfies

$$
\vec{h}_{m}+\lambda^{2} \operatorname{rot}\left(\operatorname{rot} \vec{h}_{m}\right)=0 .
$$

The magnetic field and corresponding vector potential can be written as $\vec{h}=\vec{h}_{m}+\vec{h}_{v}, \vec{A}=\vec{A}_{m}+\vec{A}_{v}$ where indexes $v$ and $m$ refer to the vortex and ferromagnet, respectively. Now, from Eq. (1i) we obtain the vortex-dipole interaction energy as

$$
F_{m v}=\frac{1}{8 \pi} \int d V\left[\operatorname{rot} \vec{h}_{m} \cdot\left(\vec{A}_{v}+\lambda^{2} \operatorname{rot} \vec{h}_{v}\right)\right]=\frac{1}{2 c} \int d V^{(i)}\left[\vec{j}_{m} \cdot \vec{\Phi}_{v}\right]+\frac{1}{2} \int d V_{v}^{(o)} \vec{A} \cdot \operatorname{rot} \vec{M}-\int d V_{v}^{(m d)} \vec{h} \cdot \vec{M}
$$

which after simple integral transformations becomes

$$
F_{m v}=\frac{1}{2 c} \int d V^{(i)}\left[\vec{j}_{m} \cdot \vec{\Phi}_{v}\right]-\frac{1}{2} \int d V^{(m d)} \vec{h}_{v} \cdot \vec{M}
$$

where $\vec{\Phi}_{v}=\left(\Phi_{\rho}, \Phi_{\varphi}, \Phi_{z}\right)=\left(0, \Phi_{0} /(2 \pi \rho), 0\right)$ denotes the vortex magnetic flux vector $\left(\Phi_{0}\right.$ is the flux quantum), and $\vec{M}$ is the magnetization of the dipole. As one can see, the interaction energy in this system consists of two parts, namely, (i) the interaction between the Meissner currents generated in the SC $\left(\vec{j}_{m}\right)$ by the MD and the vortex, and (ii) the interaction between the vortex magnetic field and the MD.

In order to obtain the value of the current induced in the superconductor by a magnetic dipole with moment $\vec{m}$ $(\vec{M}=\vec{m} \delta(x) \delta(y) \delta(z-a))$, we solve the corresponding equation for the vector potentiall 9

$$
\operatorname{rot}\left(\operatorname{rot} \vec{A}_{m}\right)+\frac{1}{\lambda^{2}} \Theta(d / 2-|z|) \vec{A}_{m}=4 \pi \operatorname{rot}(\vec{m} \delta(x) \delta(y) \delta(z-a)),
$$

where $\delta(\ldots)$ is the Dirac delta function. The results for both vector potential and magnetic field, for different orientations of the magnetic moment of the dipole, are given in the Appendix.

\section{MAGNETIC DIPOLE - VORTEX INTERACTION ENERGY}

The superconducting current induced in an infinite superconducting film with thickness $d\left(-\frac{d}{2}<z<\frac{d}{2}\right)$ by a magnetic dipole with out-of-plane magnetization, i.e. $\vec{m}=m \vec{e}_{z}$ located at $z=a$ is obtained as a solution of Eq. (4) which is given by 


$$
j_{\varphi}(\rho, z)=-\frac{c m \Phi_{0}}{2 \pi \lambda^{3}} \int_{0}^{\infty} d q \exp \left\{-q\left(|a|-\frac{d}{2}\right)\right\} q^{2} J_{1}(q \rho) C(q, z)
$$

with

$$
C(q, z)=\frac{k \cosh \left(k\left(\frac{d}{2}+z\right)\right)+q \sinh \left(k\left(\frac{d}{2}+z\right)\right)}{\left(k^{2}+q^{2}\right) \sinh (k d)+2 k q \cosh (k d)},
$$

where $k=\sqrt{1+q^{2}}, \rho=\sqrt{x^{2}+y^{2}}$, sinh and $\cosh$ denote the hyperbolic trigonometric functions and $J_{v}(x)$ is the Bessel function. For a MD placed under the SC, one should replace $z$ by $-z$ in Eq. (5b). The magnetic moment of the dipole is measured in units of $m_{0}=\Phi_{0} \lambda$, and all distances are scaled in units of $\lambda$. These units will be used in the rest of the paper. Clearly, Eq. (4) can also be used for a film of finite dimensions $L$ in the limits $\rho, a<<L$. The above integral (5a) can be solved analytically for certain asymptotic limits

$$
j_{\varphi}(\rho, z)= \begin{cases}-\frac{3 c m \Phi_{0} \rho l}{2 \pi \lambda^{3}\left(\rho^{2}+l^{2}\right)^{5 / 2}} \frac{\cosh (d / 2+z)}{\sinh (d)} & , \rho>>\max (l, 1 / d) \text { or } l>>\max (\rho, 1 / d), \\ -\frac{c m \Phi_{0}}{4 \pi \lambda^{3}}\left[\frac{\rho}{\left(\rho^{2}+l^{2}\right)^{3 / 2}}+\frac{d\left(\sqrt{\rho^{2}+l^{2}}-l\right)}{2 \rho \sqrt{\rho^{2}+l^{2}}}\right], & \rho, l<\frac{1}{d}, d<<1,\end{cases}
$$

where $l=|a|-\frac{d}{2}$ is the distance between the MD and the top surface of the SC film.

Inserting the well known expression for the magnetic field of a vortex (see Eq. (A4) in the Appendix), into Eq. (3) we find

$$
F_{m v}=\frac{m \Phi_{0}^{2}}{2 \pi \lambda} U_{\perp}\left(\rho_{v}\right)
$$

where $\rho=\rho_{v}$ denotes the position of the vortex, and

$$
U_{\perp}\left(\rho_{v}\right)=-\int_{0}^{\infty} d q \frac{q}{k(k+q \operatorname{coth}(k d / 2))} J_{0}\left(q \rho_{v}\right) \exp (-q l) .
$$

In some limiting cases it is possible to solve this integral analytically:

(1) for $d<<1$ and $\rho_{v}<1 / d$ we found: $U_{\perp}\left(\rho_{v}\right) \approx-d /\left(2\left(\rho_{v}^{2}+l^{2}\right)^{1 / 2}\right)$;

(2) for $d<1$ and a) $\rho_{v}>a$ : $U_{\perp}\left(\rho_{v}\right) \approx-d /\left(2\left(\rho_{v}^{2}+l^{2}\right)^{1 / 2}\right)+\pi d^{2}\left[H_{0}\left(\rho_{v} d / 2\right)-Y_{0}\left(\rho_{v} d / 2\right)\right] / 8$,

b) $\rho_{v}>>1 / d: U_{\perp}\left(\rho_{v}\right) \approx-2 /\left(d \rho_{v}^{3}\right)$;

(3) for $d>1$ and $\rho_{v}>>\max (l, 1 / d)$ or $l>>\max \left(\rho_{v}, 1 / d\right)$ we obtained: $U_{\perp}\left(\rho_{v}\right) \approx-l /\left(\rho_{v}^{2}+l^{2}\right)^{3 / 2}$.

Here, $H_{v}(x)$ and $Y_{v}(x)$ denote the Struve and Bessel function, respectively. One should notice that our asymptotic results for a thin SC film differ from those oiven in Eq. (7) of Ref.11 by a factor of two, and are in agreement with the calculations of Ref.15 (Eq. (3.16) of Ref.15).

In Fig. 2( $(a, b)$ the interaction energy as function of the position of the vortex are shown, for different (a) vertical positions of the magnetic dipole, and (b) thickness of the SC. Please note that the asymptotic expressions for an extremely thin SC, namely $d<<1$, give a very good description of the interaction potential (see inset of Fig. 2(b)). As expected, the problem of the interaction energy in this system is axially symmetric and this is illustrated in Fig. 2(c). In Fig. 2(a), we notice that the energy curves for different vertical positions of the dipole cross each other for large MD-vortex distances, for the case of a thick SC, namely $d>1$. Consequently, for a MD which is higher above the SC film, interaction with the vortex is stronger at large distances, i.e. $\rho_{v}>>1 / d$ (see our approximate results above, case (3)). This can be understood as follows. In the case of a thick superconductor, the magnetic field does not penetrate through the SC, and the component of the field tangential to the surface becomes important. When the MD approaches the SC surface, the value of this field component at large distances from the dipole increases, as shown in the inset of Fig. 2(a). Therefore, the interaction between the dipole and the vortex is stronger for smaller $l$ when the MD-vortex distance $\rho_{v}$ is small, and opposite, for large $\rho_{v}$, the interaction energy grows if $l$ is larger.

Naturally, the minimum of the interaction energy depicts the energetically favorable position of the vortex. Therefore, from Fig. 2 one notices that for parallel alignment of the MD magnetization and the vortex the interaction will be purely attractive, and the vortex is pinned under the MD, independently of the parameters of the system (thickness of the SC, vertical position of the MD and its magnetization strength, etc.). These parameters only determine the strength of this attractive interaction. For the anti-parallel orientation of the magnetic moment, the vortex is repelled. 
Following the same procedure, for a MD with in-plane magnetization $\left(\vec{m}=m \vec{e}_{\rho}\right.$, for $\varphi=0$, and $\vec{m}=0$ for $\varphi \neq 0$ ) we have

$$
F_{m v}=\frac{m \Phi_{0}^{2}}{2 \pi \lambda} U_{\|}\left(\rho_{v}, \varphi\right),
$$

where $\varphi$ is the angle in the SC plane between the direction of the magnetic moment of the dipole and the point of interest, and

$$
U_{\|}\left(\rho_{v}, \varphi\right)=\int_{0}^{\infty} d q \frac{q \cos \varphi}{k(k+q \operatorname{coth}(k d / 2))} J_{1}\left(q \rho_{v}\right) \exp (-q l),
$$

for which we obtain the following analytic asymptotic results

$$
U_{\|}\left(\rho_{v}, \varphi\right)= \begin{cases}\frac{\rho_{v} \cos \varphi}{\left(\rho_{v}^{2}+l^{2}\right)^{3 / 2}} & , \rho>>\max (l, 1 / d) \text { or } l>>\max (\rho, 1 / d), d>1, \\ \frac{\rho_{v} d \cos \varphi}{2 \sqrt{\rho_{v}^{2}+l^{2}}\left(l+\sqrt{\rho_{v}^{2}+l^{2}}\right)}, & d<<1 .\end{cases}
$$

In Fig. 3(a,b), the energy is plotted as function of the position of the vortex when it is moved along the direction of the MD magnetization $(\varphi=\pi)$, and Fig. 3(c) shows the complete contour plot. The vortex is attracted to one side (where the magnetic field of the MD penetrates the SC in the same direction as the vortex, see Fig. 1(b)) and repelled on the opposite side of the MD. Moreover, it is pinned in a spot whose position does not depend on the magnetization of the MD but it is influenced by the vertical position of the dipole and the thickness of the SC. When the dipole approaches the SC or the thickness of the superconductor is increased, the interaction becomes stronger and the vortex will move closer to the MD.

From our asymptotic expressions for the interaction energy the most favorable position of the vortex can be easily obtained by minimizing the energy. In the thin film approximation $(d<<1)$, we obtain $\rho_{v}^{*}=\sqrt{2+2 \sqrt{5}} l / 2 \approx 1.272 l$, while for $d>1$, but $l>>1 / d$ we have $\rho_{v}^{*}=l / \sqrt{2} \approx 0.7071 l$. In Fig. $($ (a) we show the $d$-dependence of the equilibrium position as function of the thickness of the SC. The $\rho_{v}^{*}(l)$ function is in general non-linear, as shown in Fig. A(b). A peculiar fact is that for $d>2$, the energetically preferable position of the vortex depends only on $l$. This follows from the fact that the magnetic field of the dipole penetrates only in the surface layer of the superconductor down to the penetration depth $\lambda$ so that further increase of the SC thickness does not affect the total interaction. From Fig. A(b) one should notice that our asymptotic results describe nicely the behavior of the system for $d<<1$ or $l>>d$, when $d>1$.

For the case of in-plane MD, it should be emphasized that the vortex is attracted to the side of the MD where the magnetic field is parallel to the field of the vortex, which is similar to the case of the out-of-plane magnetized dipole. This conclusion also follows from the observation of the interaction between the induced currents and the vortex. For in-plane magnetized MD above the superconductor, the components of the superconducting current in the absence of vortices are given by

$$
\begin{gathered}
j_{\rho}(\rho, \varphi, z)=-\frac{c m \Phi_{0} \sin \varphi}{2 \pi \lambda^{3} \rho} \int_{0}^{\infty} d q \exp (-q l) q J_{1}(q \rho) C(q, z), \\
j_{\varphi}(\rho, \varphi, z)=\frac{c m \Phi_{0} \cos \varphi}{2 \pi \lambda^{3}} \int_{0}^{\infty} d q \exp (-q l) q^{2}\left(\frac{J_{1}(q \rho)}{q \rho}-J_{0}(q \rho)\right) C(q, z), \\
j_{z}(\rho, \varphi, z)=0 .
\end{gathered}
$$

We obtained the following asymptotic behavior of these components:

a) $\rho>>\max (l, 1 / d)$ or $l>>\max (\rho, 1 / d)$

$$
\begin{aligned}
& j_{\rho}(\rho, \varphi, z)=-\frac{c m \Phi_{0} \sin \varphi}{2 \pi \lambda^{3}\left(\rho^{2}+l^{2}\right)^{3 / 2}} \frac{\cosh (d / 2+z)}{\sinh (d)}, \\
& j_{\varphi}(\rho, \varphi, z)=\frac{c m \Phi_{0} \cos \varphi}{2 \pi \lambda^{3}\left(\rho^{2}+l^{2}\right)^{3 / 2}} \frac{2 \rho^{2}-l^{2}}{\rho^{2}+l^{2}} \frac{\cosh (d / 2+z)}{\sinh (d)},
\end{aligned}
$$


b) $\rho, l<\frac{1}{d}, d<<1$

$$
\begin{aligned}
& j_{\rho}(\rho, \varphi, z)=-\frac{c m \Phi_{0} \sin \varphi}{4 \pi \lambda^{3}} \frac{\sqrt{\rho^{2}+l^{2}}-l}{\rho^{2} \sqrt{\rho^{2}+l^{2}}}, \\
& j_{\varphi}(\rho, \varphi, z)=\frac{c m \Phi_{0} \cos \varphi}{4 \pi \lambda^{3}}\left[\frac{\sqrt{\rho^{2}+l^{2}}-l}{\rho^{2} \sqrt{\rho^{2}+l^{2}}}-\frac{l}{\left(\rho^{2}+l^{2}\right)^{3 / 2}}\right] .
\end{aligned}
$$

For the case of the MD under the SC, these currents change sign. The detailed expressions for the magnetic fields and the vector potential as solution of Eq. (4) are given in the Appendix. The vector plots of the screening currents for both directions of the MD magnetization are presented in Fig. 0 .

Previous theoretical studie 20 on the interaction of a magnetic moment and a vortex used a Gibbs free energy argument to show that this force was attractive. In our case, the pinning force between a vortex and the MD consists of two parts: (i) the interaction between the vortex fringing field and the magnetic moment, and (ii) the interaction between the vortex and the screening currents created in response to the magnetic dipole field. One should notice (Fig. F(a)) that although the magnetic field of a MD with out-of plane magnetization changes sign in the SC plane, the superconducting screening current is always in the same direction, and therefore, the interaction between the MD and a vortex with parallel orientation of the field is attractive (consequently, the vortex will sit under the MD), and analogously, for anti-parallel orientation of the magnetic fields they repel each other.

For the case of a MD with in-plane magnetization, the Meissner current vector plot in Fig. 5(b) shows that the direction of the current is such that the vortex is attracted to the region where the magnetic field of a MD is parallel to it and repelled on the other side of a MD due to the opposite direction of the current.

\section{PINNING OF MULTIPLE VORTICES AND/OR ANTI-VORTICES}

\section{A. In-plane magnetized dipole}

Obviously, in the case of in-plane magnetization, the vortex will be repelled on the side of the MD to which the magnetic moment points to and pinned on the opposite side (and vice versa for the antivortex). The case when the dipole itself creates vortices was experimentally investigated by Van Bael et al.18, where it was found that the stray field of the in-plane magnetic dipole induces a vortex-antivortex pair (VA) at the poles, at positions predicted by our analysis (vortex towards negative pole, antivortex towards positive pole). Therefore, this asymmetric pinning potential provides stability for vortex-antivortex configurations. One would expect that the position of the pinning sites depends on the magnetic moment of the MD and the number of flux quanta carried by each vortex. To determine the preferable position of the vortex-antivortex pair in the presence of an in-plane MD we put the vortex at $\left(\rho_{v}, \varphi_{v}\right)$ and the antivortex at $\left(\rho_{a v}, \varphi_{a v}\right)$ with respect to the MD and calculate their interaction with the dipole. The vortexantivortex interaction energy is given by 26

$$
F_{v-a v}\left(R, L_{v}, L_{a v}\right)=-\frac{L_{v} L_{a v} \Phi_{0}^{2}}{8 \pi^{2} \lambda}\left(d K_{0}(R)+2 \int_{0}^{\infty} d q \frac{J_{0}(q R)}{k^{2} Q}\right),
$$

where $R=\sqrt{\rho_{v}^{2}+\rho_{a v}^{2}-2 \rho_{v} \rho_{a v} \cos \left(\varphi_{v}-\varphi_{a v}\right)}$ is the distance between the vortex and antivortex, $K_{0}(x)$ is the MacDonald function, and $L_{v}, L_{a v}$ are the vorticity of the vortex and anti-vortex, respectively.

First, let us suppose that the vortex-antivortex pair appears due to the stray field of the dipole. In that case, this pair nucleates where the superconducting current is maximal, namely, under the dipole (Fig. F(b)), with vortex and antivortex situated on opposite sides of the dipole. Therefore, due to the symmetry of the applied potential (Fig. 3(c)), we may assume that $\rho_{v}=\rho_{a v}$, and $\varphi_{v}=\pi-\varphi_{a v}$. To investigate the stability of such a pair of vortices, we calculate the total interaction energy in this system with respect to the position of the vortices. The results are shown as contourplots in Figs. 6(a-c) for different values of the magnetic moment of the dipole. The lowest value of the interaction energy gives the energetically favorable position of the vortex-antivortex pair [apart from the global minimum at $x=0$ (zero distance between vortices), where the energy equals $-\infty]$. Obviously, the lateral motion of vortices is forbidden by the high energy barriers, while along the direction of the dipole, also an energy barrier exists, but lower than the lateral ones, showing us the preferable direction of possible vortex-antivortex annihilation.

Knowing this, we put the vortex and antivortex along the direction of the MD, in such a way that $\rho_{v}=\rho_{a v}$, and $\varphi_{v}=\pi, \varphi_{a v}=0$, and investigate the pinning potential as function of the distance of the vortex and antivortex from 
the MD. We substitute $R=2 \rho_{v}$ in Eq. (12) and add this energy to the values of the MD-vortex and MD-antivortex attraction energy obtained from Eq. 8a). The result is shown in Fig. 7 for (a) different values of the vorticity $\left(L_{v}\right.$, $L_{a v}$ ), and (b) different magnetization and $L_{v}=L_{a v}=1$. After minimization of the total energy over $\rho_{v}$, we obtain the position of the vortex-antivortex pair. In the thin superconductor case $(d<1)$ this leads to the non-linear equation

$$
\frac{2 \pi m \rho_{v}}{\sqrt{l^{2}+\rho_{v}^{2}}\left(l+\sqrt{l^{2}+\rho_{v}^{2}}\right)}\left[1-\rho_{v}^{2}\left(\frac{1}{l^{2}+\rho_{v}^{2}}+\frac{1}{\sqrt{l^{2}+\rho_{v}^{2}}\left(l+\sqrt{l^{2}+\rho_{v}^{2}}\right)}\right)\right]=\frac{L_{v} L_{a v}}{L_{v}+L_{a v}} .
$$

Although all forces acting in the system are attractive, it is clearly visible that annihilation of vortices can be prevented by their strong confinement at the poles of the MD (the position of vortex-antivortex pairs is illustrated by the open circles in Fig. 7). The interaction energy shows an absolute minimum for $\rho_{v}=0$, implying the annihilation, but in order to do that vortices have to cross a potential barrier. Therefore, the vortex-antivortex configuration can be stable in this system. To determine if this vortex state is metastable or the ground state one should compare its total energy to the one without vortices. In that case, the existence of the pairs as the ground state will not depend only on the magnetization of the dipole but also on the parameters of the superconductor (i.e. $\lambda, \xi)$ which influence the equilibrium phase diagram via the self-energy of the vortices.

From Fig. 月(b) it is clear that in the case of $L_{v}=L_{a v}=1$ there exists a critical MD magnetization for which the annihilation barrier disappears. More generally, the critical value of the magnetic moment of the MD, in the thin SC film limit, follows from Eq. (13) (by the condition that the function has no solution) and is given by

$$
m^{*}=1.08529 \frac{L_{v} L_{a v} l}{L_{v}+L_{a v}}
$$

From this equation the critical values of the vorticity, when attraction becomes stronger than the pinning, can also be estimated. For $L_{v}=L_{a v}$ we have $m^{*}=1.08529 L_{v} l / 2$ and using the fact that $\Phi^{+} / \Phi_{0}=2 m / l$ for an in-plane MD we obtain the critical condition $\Phi^{+} / \Phi_{0}=1.08529 L_{v}$, where $\Phi^{+}$denotes the flux through the region of the positive stray field of the dipole at the SC surface. This implies that there is a connection between the appearance of stable vortex-antivortex pairs and the amount of penetrating flux of the magnetic dipole. Notice that the critical $\Phi^{+}$is not exactly quantized in units of $\Phi_{0}$ which is a mesoscopic effect.22 The quantization condition $\Phi=\oint_{C} \vec{A} \cdot d \vec{l}=L \Phi_{0}$ cannot be used because it is not possible to construct a contour $C$ around the positive stray field region where the current is zero (see Fig. 5(b)). The inhomogeneous magnetic field of the dipole stimulates the creation of a vortex and an antivortex at opposite poles. These vortices cannot be treated independently and it turns out that larger flux is needed in order not only to create this pair but also to keep them apart.

As shown above, for the case of the experimentally important thin SC film approximation, the position of the vortex-antivortex pair with respect to the dipole can be obtained from Eq. (13). This position depends both on the vertical position of the dipole and its magnetization strength. For fixed magnetic moment of the dipole, the vortex-antivortex pair can only be stabilized for $l$ below some critical value which is obtained from Eq. (14). If we put $m=m^{*}$ in Eq. (13), we obtain the simple dependence $\rho_{v}^{*}=0.4904 l^{*}$. The dependence of $\rho_{v}^{*}$ on $l$ is shown in Fig. 8, for different values of the magnetization, where also the stability region of the vortex-antivortex pair is indicated. One can see three regions in this diagram: i) the region where the vortex-antivortex pair is unstable, which is bounded by $\rho_{v}^{*}=0.4904 l$, for any value of the magnetization of the MD, ii) the region of stability, and iii) the forbidden area, for $\rho_{v}^{*}>1.272 l$. The latter condition follows from Sec. III (see Fig. 団(b)). Notice that with increasing distance between the dipole and the SC film, the pinning sites move further from the center of the dipole, up to a certain point when the interaction between vortices overwhelms the pinning force. After that, the distance between vortex and antivortex decreases and they finally annihilate. The maximum distance $\rho_{v}^{*}$ follows from Eq. (13) by taking the derivative with respect to $l$ and leads to $\rho_{v}^{*} \max =0.7071 l_{\max }$ (open dots in Fig. 8), which again corresponds to the single vortex situation for $l>d$ (see Fig. 价).

\section{B. Out-of-plane magnetized dipole}

Due to the strong field inhomogeneity and the reversal of the direction of the magnetic field in the vicinity of the MD with out-of plane magnetization, and the fact that the net magnetic flux due to the MD in the SC plane equals zero one expects that vortex-antivortex configurations might be stable in such applied field, as predicted earlier in Ref. 33 for a finite size superconductor. As shown in Fig. 2, an individual vortex is strongly attracted by the MD and the antivortices are repelled. Therefore we artificially put one vortex with vorticity $L_{v}$ under the MD and assume the existence of a ring of $n_{a v}$ single antivortices around it, with radius $\rho_{v} .23$ Adding the interaction between each two vortices to our previous expression for the interaction energy, we obtain the total interaction energy 


$$
F_{\text {int }}=\frac{m \Phi_{0}^{2}}{2 \pi \lambda}\left(L_{v} U_{\perp}(0)-n_{a v} U_{\perp}\left(\rho_{v}\right)\right)+n_{a v} F_{v-a v}\left(\rho_{v}, L_{v}, 1\right)-\frac{n_{a v}}{2} \sum_{j=1}^{n_{a v}-1} F_{v-a v}\left(\rho_{v} \sqrt{2-2 \cos \frac{2 \pi j}{n_{a v}}}, 1,1\right),
$$

where the first two terms describe the dipole-vortex and dipole-antivortex interactions, the third term is the vortexantivortex attraction term, and the last term is the repulsion energy between antivortices. The function $F_{v-a v}$ is given by Eq. (12). Although the vortex is attracted by the antivortices, annihilation is prevented by the repulsion between the antivortex and the MD (for example, see Fig. 9, for $L_{v}=n_{v}=1$ ). Naturally, this energy barrier becomes smaller with decreasing magnetic moment of the dipole. Nevertheless, if a barrier exists, the interaction energy shows one local minimum, meaning that antivortices would not be repelled to infinity but to a certain point. The position of this local minimum we obtained in the same manner as previously, which within the thin film approximation, leads to the equation

$$
\frac{4 \pi m \rho_{v}^{2}}{\left(l^{2}+\rho_{v}^{2}\right)^{3 / 2}}=2 L_{v}-n_{a v}+1 .
$$

From this expression, the threshold value of the magnetic moment (when the potential barrier appears) is obtained as

$$
m^{*}=\left(\frac{3}{4}\right)^{3 / 2} \frac{\left(2 L_{v}-n_{a v}+1\right) l}{\pi} .
$$

For lower values of the magnetic moment, the energetic barrier between vortices disappears and annihilation can not be prevented. Analogously, for fixed magnetic moment, increasing vorticity $L_{v}$ of the vortex will make the attraction stronger and for a certain value of $L_{v}$, antivortices will be able to overcome the barrier. This critical value of vorticity can also be estimated from Eq. (17). One should notice that we leave the possibility of $L_{v} \neq n_{a v}$, which corresponds to the experimental situation when first positive external flux lines are pinned by the magnetic center, and then the polarity of the applied field is changed. Using $\Phi^{+} / \Phi_{0}=4 \sqrt{3} \pi / 9 \mathrm{~m} / \mathrm{l}$ for out-of-plane magnetic dipole polarization, we find for $m=m^{*}$ that the critical condition becomes $\Phi^{+} / \Phi_{0}=\left(2 L_{v}-n_{a v}+1\right) / 2$, where $\Phi^{+}$denotes the flux through the region of positive stray field of the dipole. One should notice that the first stable vortex-antivortex pair $\left(L_{v}=n_{a v}=1\right)$ appears for $\Phi^{+}=\Phi_{0}$, and further increase of vorticity is a quantized process, with $\Delta \Phi^{+}=\Phi_{0} / 2$ necessary for stability for each one-unit-increase of $L_{v}$ and $n_{a v}$. Also in this case we cannot define a path around the positive stray field region where the superconducting current is zero (see Fig. 5(a)) and consequently the flux quantization condition does not apply here.

In this treatment, we assumed the presence of a giant vortex under the dipole as an energetically preferable state. It is well known that in infinite superconductors, thus in the absence of boundaries imposing the symmetry of the superconducting state, the giant vortex splits into multivortices. Since in our case an inhomogeneous applied magnetic field dictates the behavior of superconducting electrons, it is not clear which state carries less energy. Which central vortex configuration is realized depends on the parameters of the superconductor, i.e. $\xi, \lambda$ and $d$, which come into play through the self energy of the involved vortices. Therefore, for a particular superconducting film, we extend our approach to the case of multivortices surrounded by an multi-antivortex ring. Namely, we investigate the stability of $N$ vortex-antivortex pairs symmetrically arranged around the dipole, where vortices sit on a ring with radius $\rho_{v}$, and antivortices occupy the corresponding positions on the ring with radius $\rho_{a v}$. We apply the same approach as before, calculating the interaction energy in a similar manner as in Eq. (15), where the interaction between each two vortices is included. After minimization of the interaction energy with respect to the parameters $\rho_{v}$ and $\rho_{a v}$, we obtain numerically the energetically favorable positions of the vortices. The results are shown in Fig. 10, for the case of $N=3$ (see inset of Fig. 10). Increasing magnetization of the dipole increases the distance between vortices and antivortices in a way that vortices come closer to the dipole and antivortices are repelled further away. It should be noted that a certain critical value of the magnetization is needed to prevent annihilation. In Fig. 11 we give this threshold value as function of $l$ for different values of $N$. One should note that this value of magnetic moment implies again the quantization of the penetrating flux (as in the case of a giant vortex surrounded by antivortices), but with $\Delta \Phi^{+}=1.0489 \Phi_{0}$ necessary for stability of the first pair, and slightly decreasing for additional pairs. For $N>4, \Delta \Phi^{+}$ becomes smaller than the flux quantum. The interpretation of this behavior can be that multivortices eventually join into a giant vortex, or that a different geometry of the superconducting state appears. For example, for larger number of pairs, due to the large linear density of vortices along the ring, they could rearrange, forming more than a single ring.

Fig. 11 gives only the critical condition for stability of vortex-antivortex pairs. However, which configuration has the lowest energy and is thus the energetically preferable state cannot be inferred from this figure. In order to compare 
the energies of states with different number of pairs, i.e. $N$, we include the self-energy of individual vortices in the calculation

$$
F_{v}=\frac{\Phi_{0}^{2}}{16 \pi^{2} \lambda}\left[d \ln \frac{\lambda}{\xi}+2 \tanh \left(\frac{d}{2}\right) \ln \left(1+\operatorname{cotanh}\left(\frac{d}{2}\right)\right)\right]
$$

The numerical results, as referred to the Meissner state, are shown in Fig. 12, for a thin SC film with $\lambda / \xi=10$. With increasing magnetic moment of the dipole the energetically favorable state goes through successive states in which $N$ increases. If we calculate again the flux $\Delta \Phi^{+}$which now corresponds to the appearance of the next $N$ state as the ground state, we find that $\Delta \Phi^{+}=1.97 \Phi_{0}$, for the appearance of the first pair, and for $N \geq 2$, the additional flux slightly decreases with increasing $N$, starting from $\Delta \Phi^{+}=1.089 \Phi_{0}$, for $N=2$. Larger flux needed for the first vortex-antivortex state can be explained by the fact that this cylindrically asymmetrical state appears in a symmetrical magnetic potential. For $N \geq 2$, the results correspond to our previous analysis. It should be stressed that these results depend on the parameters of the superconductor, namely $\lambda$ and $\xi$, which come into play through the self-energy of the vortices. This is in contrast to the interaction energy (see the inset of Fig. 12) which, in the London approximation, is independent of $\xi$.

\section{CONCLUSION}

To summarize, we applied the London theory to investigate flux pinning in SC films due to the presence of a magnetic dipole situated above (or under) the SC. Depending on the direction of the dipole magnetic moment, we obtained exact analytic expressions for the MD-vortex interaction energy and screening currents. We obtained the asymptotic behavior of the interaction potential and the induced currents for specific values of the involved parameters. We calculated the pinning potential for both an in- and out-of-plane magnetized dipole. Our results show that an out-of-plane magnetized dipole attracts a vortex if aligned parallel to it, and opposite, for anti-parallel alignment the vortex is repelled. This is a consequence of the mono-directional superconducting current induced in the SC for outof-plane magnetization of the MD. However, for in-plane magnetization, the dipole-vortex interaction shows a dual behavior, namely, the vortex is attracted to the negative pole of the MD and repelled on the other side. Moreover, the position of the pinning site depends on the position of the MD and thickness of the SC. We calculated these dependences and showed that it is linear for thin superconductors, or large MD-vortex distances. Due to the dual behavior of the pinning potential, we explored the possible co-existence of vortices and antivortices in such systems. The total interaction energy calculation leads to the conclusion that the vortex and antivortex are separated by an energy barrier due to the short range interaction with the dipole, and therefore, these pairs could be stable. Both inand out-of-plane magnetized dipoles are able to keep these vortices apart. We calculated analytically the interaction potential in the presence of vortex-antivortex pairs (or giant vortex-single antivortices) and gave estimates of the parameters necessary for stability of such fascinating configurations in a thin SC film.

\section{ACKNOWLEDGMENTS}

This work was supported by the Flemish Science Foundation (FWO-Vl), the Belgian Inter-University Attraction Poles (IUAP), the "Onderzoeksraad van de Universiteit Antwerpen" (GOA), and the ESF programme on "Vortex matter". Stimulating discussions with D. Vodolazov, V. V. Moshchalkov, and M. Van Bael are gratefully acknowledged.

\section{APPENDIX: THE MAGNETIC FIELD OF A MAGNETIC DIPOLE IN THE PRESENCE OF A SC FILM}

The magnetic field of a vortex $\vec{h}_{v}=\operatorname{rot} \vec{A}_{v}$, which is perpendicular to the plane of the film, is determined as the solution of the system of equations

$$
\vec{A}_{v}(\vec{\rho}, z)+\lambda^{2} \operatorname{rot} \operatorname{rot} \vec{A}_{v}(\vec{\rho}, z)=\vec{\Phi}\left(\vec{\rho}-\vec{\rho}_{v}\right), \quad|z|<d / 2,
$$




$$
-\nabla^{2} \vec{A}_{v}(\vec{\rho}, z)=0 \quad|z|>d / 2
$$

with the following boundary conditions: i) the continuity of the vector potential components at $z= \pm d / 2$, and ii) their vanishing far from the superconductor (at $|z| \rightarrow \infty)$. Here $\vec{\Phi}\left(\vec{\rho}-\vec{\rho}_{v}\right)$ takes into account the vortex, where $\vec{\rho}_{v}$ is the vortex position in the film plane. In cylindrical coordinates $\Phi_{\rho}=\Phi_{z}=0, \Phi_{\varphi}=L \Phi_{0} / 2 \pi\left|\vec{\rho}-\vec{\rho}_{v}\right|$ (so that $\left.\operatorname{rot} \vec{\Phi}=L \Phi_{0} \delta\left(\vec{\rho}-\vec{\rho}_{v}\right)\right)$. The solutions of Eqs. A1a)-(A1b) are25,26

$$
\begin{gathered}
A_{v \varphi}^{(i)}(\vec{\rho}, z)=\frac{L \Phi_{0}}{2 \pi \lambda} \int_{0}^{\infty} d q \frac{J_{1}(q R / \lambda)}{k^{2}}\left[1-\frac{k q \cosh (k z / \lambda)}{Q \sinh (k d / 2 \lambda)}\right], \\
A_{v \varphi}^{(o)}(\vec{\rho}, z)=\frac{L \Phi_{0}}{2 \pi \lambda} \int_{0}^{\infty} d q \frac{J_{1}(q R / \lambda)}{Q} \exp \left(-q \frac{2|z|-d}{2 \lambda}\right) .
\end{gathered}
$$

Here $k=\left(1+q^{2}\right)^{1 / 2}, Q=k[k+q \operatorname{coth}(k d / 2 \lambda)], \quad R=\left|\vec{\rho}-\vec{\rho}_{v}\right|=\left[\rho^{2}+\rho_{v}^{2}-2 \rho \rho_{v} \cos \left(\varphi-\varphi_{v}\right)\right]^{1 / 2}, J_{\nu}(x)$ is the Bessel function, index " $i(o)$ " denotes the field inside (outside) the superconductor. The components of the vortex magnetic field are given by 26

$$
\begin{gathered}
h_{v z}^{(i)}(\rho, z)=\frac{L \Phi_{0}}{2 \pi \lambda^{2}}\left[K_{0}\left(\frac{R}{\lambda}\right)-\int_{0}^{\infty} \frac{d q q^{2}}{k Q} J_{0}\left(\frac{q R}{\lambda}\right) \frac{\cosh (k z / \lambda)}{\sinh (k d / 2 \lambda)}\right], \\
h_{v \rho}^{(i)}(\rho, z)=\frac{L \Phi_{0}}{2 \pi \lambda^{2}} \int_{0}^{\infty} \frac{d q q}{Q} J_{1}\left(\frac{q R}{\lambda}\right) \frac{\sinh (k z / \lambda)}{\sinh (k d / 2 \lambda)}, \\
h_{v z}^{(o)}(\rho, z)=\frac{L \Phi_{0}}{2 \pi \lambda^{2}} \int_{0}^{\infty} \frac{d q q}{Q} J_{0}\left(\frac{q R}{\lambda}\right) \exp \left(-q \frac{2|z|-d}{2 \lambda}\right), \\
h_{v \rho}^{(o)}(\rho, z)=\frac{L \Phi_{0}}{2 \pi \lambda^{2}} \operatorname{sgn}(z) \int_{0}^{\infty} \frac{d q q}{Q} J_{1}\left(\frac{q R}{\lambda}\right) \exp \left(-q \frac{2|z|-d}{2 \lambda}\right), \\
h_{v \varphi}^{(i)}=h_{v \varphi}^{(o)}=0,
\end{gathered}
$$

where $K_{0}(x)$ is the MacDonald function.

The magnetic field of a dipole, situated at thedistance $l$ above the film, can be written in the following way (here and below $l=|a|-d / 2$ ) (see, for example, Ref.27)

$$
\vec{h}_{m}(\vec{\rho}, z)=\left\{\begin{array}{lc}
\vec{h}_{d}(\vec{\rho}, z)+\vec{h}_{m,+}^{(o)}(\vec{\rho}, z), & z>d / 2, \\
\vec{h}_{m}^{(i)}(\vec{\rho}, z), & -d / 2 \leq z \leq d / 2, \\
\vec{h}_{m,-}^{(o)}(\vec{\rho}, z), & z<-d / 2,
\end{array}\right.
$$

where $\vec{h}_{d}(\vec{\rho}, z)$ is the direct contribution from the dipole, $\vec{h}_{m,+}^{(o)}(\vec{\rho}, z)$ is the induced field above the superconductor, $\vec{h}_{m}^{(i)}(\vec{\rho})$ is the dipole field penetrating inside the superconductor and $\vec{h}_{m,-}^{(o)}(\vec{\rho})$ denotes the field of the dipole below the film. The vector potential $\vec{A}_{d}(\vec{\rho}, z)$ is defined by the equation

$$
\operatorname{rot} \vec{A}_{d}(\vec{\rho}, z)=4 \pi \vec{m} \delta(\vec{\rho}) \delta(z-a),
$$

where $\vec{m}$ is the magnetic moment of the dipole. The potential $\vec{A}_{m}^{(i)}(\vec{\rho})$ is found as the solution of Eq. A1a) with zero right side and $\vec{A}_{m, \pm}^{(o)}(\vec{\rho})$ as solution of Eq. (A1b). The integration constants have to be found through similar boundary 
conditions as for the vortex field distribution: i) the continuity of the vector potential components at $z= \pm d / 2$ and ii) their vanishing at $|z| \rightarrow \infty$.

We consider two orientations of the MD magnetization.

I) The dipole is directed perpendicular to the film plane, i.e. $m_{\rho}=m_{\varphi}=0, m_{z}=m$. In this case the vector potential has only an azimuthal component and is described by the following expressions:

$$
\begin{gathered}
A_{d \varphi}(\rho, z)=\frac{m}{\lambda^{2}} \int_{0}^{\infty} d q q J_{1}\left(\frac{q \rho}{\lambda}\right) \exp \left(-\frac{q}{\lambda}|z-a|\right)=\frac{m \rho}{\left[\rho^{2}+(z-a)^{2}\right]^{3 / 2}}, \\
A_{m \varphi}^{(i)}(\rho, z)=\frac{1}{\lambda^{2}} \int_{0}^{\infty} d q q J_{1}\left(\frac{q \rho}{\lambda}\right)\left[B_{1}(q) \exp \left(-\frac{k z}{\lambda}\right)+D_{1}(q) \exp \left(\frac{k z}{\lambda}\right)\right], \\
A_{m \varphi, \pm}^{(o)}(\rho, z)=\frac{1}{\lambda^{2}} \int_{0}^{\infty} d q q J_{1}\left(\frac{q \rho}{\lambda}\right) B_{2, \pm}(q) \exp \left(-\frac{q z}{\lambda}\right),
\end{gathered}
$$

where $B_{1}(q)=B(q) q(k-q) \exp (-k d / 2 \lambda), \quad D_{1}(q)=B(q) q(k+q) \exp (k d / 2 \lambda), \quad B_{2,+}(q) \quad=$ $-B(q) \sinh (k d / \lambda) \exp (q d / 2 \lambda), \quad$ and $\quad B_{2,-}(q) \quad=\quad 2 B(q) k q \exp (q d / 2 \lambda), \quad$ with $B(q)=$ $2 m \exp (-q l / \lambda) /\left((k+q)^{2} \exp (k d / \lambda)-(k-q)^{2} \exp (-k d / \lambda)\right)$.

Consequently, we obtain the following components of the dipole magnetic field

$$
\begin{gathered}
h_{m z}^{(i)}(\rho, z)=\frac{1}{\lambda^{3}} \int_{0}^{\infty} d q q^{2} J_{0}\left(\frac{q \rho}{\lambda}\right)\left[B_{1}(q) \exp \left(-\frac{k z}{\lambda}\right)+D_{1}(q) \exp \left(\frac{k z}{\lambda}\right)\right] \\
h_{m \rho}^{(i)}(\rho, z)=\frac{1}{\lambda^{3}} \int_{0}^{\infty} d q k q J_{1}\left(\frac{q \rho}{\lambda}\right)\left[B_{1}(q) \exp \left(-\frac{k z}{\lambda}\right)-D_{1}(q) \exp \left(\frac{k z}{\lambda}\right)\right] \\
h_{d z}(\rho, z)=\frac{m}{\lambda^{3}} \int_{0}^{\infty} d q q^{2} J_{0}\left(\frac{q \rho}{\lambda}\right) \exp \left(-\frac{q}{\lambda}|z-a|\right)=\frac{m\left[2(z-a)^{2}-\rho^{2}\right]}{\left[\rho^{2}+(z-a)^{2}\right]^{5 / 2}} \\
h_{d \rho}(\rho, z)=\frac{m \operatorname{sgn}(z-a)}{\lambda^{3}} \int_{0}^{\infty} d q q^{2} J_{1}\left(\frac{q \rho}{\lambda}\right) \exp \left(-\frac{q}{\lambda}|z-a|\right)=\frac{3 m \rho(z-a)}{\left[\rho^{2}+(z-a)^{2}\right]^{5 / 2}}, \\
h_{m z, \pm}^{(o)}(\rho, z)=\frac{1}{\lambda^{3}} \int_{0}^{\infty} d q q^{2} J_{0}\left(\frac{q \rho}{\lambda}\right) B_{2, \pm}(q) \exp \left(-\frac{q z}{\lambda}\right) \\
h_{m \rho, \pm}^{(o)}(\rho, z)=\frac{1}{\lambda^{3}} \int_{0}^{\infty} d q q^{2} J_{1}\left(\frac{q \rho}{\lambda}\right) B_{2, \pm}(q) \exp \left(-\frac{q z}{\lambda}\right) \\
h_{m \varphi}^{(1)}=h_{m \varphi, \pm}^{(2)}=0 .
\end{gathered}
$$

II) The dipole is magnetized in the direction parallel to the SC film plane (in-plane magnetization). Following the same procedure as above, we obtain

$$
h_{d \rho}(\rho, \varphi, z)=\frac{m \cos \varphi}{2 \lambda^{3}} \int_{0}^{\infty} d q q^{2} \exp \left(-\frac{q}{\lambda}|z-a|\right)\left[J_{2}\left(\frac{q \rho}{\lambda}\right)-J_{0}\left(\frac{q \rho}{\lambda}\right)\right]=\frac{m\left[2 \rho^{2}-(z-a)^{2}\right] \cos \varphi}{\left[\rho^{2}+(z-a)^{2}\right]^{5 / 2}}
$$




$$
\begin{aligned}
& h_{d \varphi}(\rho, \varphi, z)=\frac{m \sin \varphi}{\lambda^{2} \rho} \int_{0}^{\infty} d q q \exp \left(-\frac{q}{\lambda}|z-a|\right) J_{1}\left(\frac{q \rho}{\lambda}\right)=\frac{m \sin \varphi}{\left[\rho^{2}+(z-a)^{2}\right]^{3 / 2}} \\
& h_{d z}(\rho, \varphi, z)=-\frac{m \cos \varphi}{\lambda^{3}} \int_{0}^{\infty} d q q^{2} \exp \left(-\frac{q}{\lambda}|z-a|\right) J_{1}\left(\frac{q \rho}{\lambda}\right)=-\frac{3 m \rho|z-a| \cos \varphi}{\left[\rho^{2}+(z-a)^{2}\right]^{5 / 2}} \\
& h_{m \rho}^{(i)}(\rho, \varphi, z)=\frac{\cos \varphi}{2 \lambda^{3}} \int_{0}^{\infty} d q k q\left[J_{2}\left(\frac{q \rho}{\lambda}\right)-J_{0}\left(\frac{q \rho}{\lambda}\right)\right] \\
& \times\left[D_{1}(q) \exp \left(\frac{k z}{\lambda}\right)-B_{1}(q) \exp \left(-\frac{k z}{\lambda}\right)\right] \text {, } \\
& h_{m \varphi}^{(i)}(\rho, \varphi, z)=\frac{\sin \varphi}{\lambda^{2} \rho} \int_{0}^{\infty} d q k J_{1}\left(\frac{q \rho}{\lambda}\right)\left[D_{1}(q) \exp \left(\frac{k z}{\lambda}\right)-B_{1}(q) \exp \left(-\frac{k z}{\lambda}\right)\right] \\
& h_{m z}^{(i)}(\rho, \varphi, z)=-\frac{\cos \varphi}{\lambda^{3}} \int_{0}^{\infty} d q q^{2} J_{1}\left(\frac{q \rho}{\lambda}\right)\left[D_{1}(q) \exp \left(\frac{k z}{\lambda}\right)+B_{1}(q) \exp \left(-\frac{k z}{\lambda}\right)\right], \\
& h_{m \rho, \pm}^{(o)}(\rho, \varphi, z)=-\frac{\cos \varphi}{2 \lambda^{3}} \int_{0}^{\infty} d q q^{2} \exp \left(-\frac{q z}{\lambda}\right)\left[J_{2}\left(\frac{q \rho}{\lambda}\right)-J_{0}\left(\frac{q \rho}{\lambda}\right)\right] B_{2, \pm}(q) \\
& h_{m \varphi, \pm}^{(o)}(\rho, \varphi, z)=-\frac{\sin \varphi}{\lambda^{2} \rho} \int_{0}^{\infty} d q q \exp \left(-\frac{q z}{\lambda}\right) J_{1}\left(\frac{q \rho}{\lambda}\right) B_{2, \pm}(q), \\
& h_{m z, \pm}^{(o)}(\rho, \varphi, z)=-\frac{\cos \varphi}{\lambda^{3}} \int_{0}^{\infty} d q q^{2} \exp \left(-\frac{q z}{\lambda}\right) J_{1}\left(\frac{q \rho}{\lambda}\right) B_{2, \pm}(q)
\end{aligned}
$$

* Permanent address: Donetsk Physical \& Technical Institute, National Academy of Sciences of Ukraine, Donetsk 83114, Ukraine

\# Electronic address: peeters@uia.ua.ac.be

${ }^{1}$ V. V. Shmidt, Sov. Phys.- JETP 30, 1137 (1970).

${ }^{2}$ M. Baert, V.V. Metlushko, R. Jonckheere, V.V. Moshchalkov, and Y. Bruynseraede, Phys. Rev. Lett. 74, 3269 (1995).

${ }^{3}$ L. N. Bulaevskii, E. M. Chudnovsky, and M. P. Maley, Appl. Phys. Lett. 76, 2594 (2000).

${ }^{4}$ J. I. Martin, M. Velez, J. Nogues, and I. K. Schuller, Phys. Rev. Lett. 79, 1929 (1997).

${ }^{5}$ M. J. Van Bael, L. Van Look, K. Temst, M. Lange, J. Bekaert, G. Guentherodt, V. V. Moshchalkov, and Y. Bruynseraede, Physica C 332, 12 (2000).

${ }^{6}$ M. Lange, M. J. Van Bael, L. Van Look, K. Temst, J. Swerts, G. Guentherodt, V. V. Moshchalkov, and Y. Bruynseraede, Europhys. Lett. 53, 646 (2001).

${ }^{7}$ I. F. Lyuksyutov and V. L. Pokrovsky, Phys. Rev. Lett. 81, 2344 (1998).

${ }^{8}$ I. F. Lyuksyutov and V. L. Pokrovsky, cond-mat/9903312.

${ }^{9}$ S. Erdin, A. F. Kayali, I. F. Lyuksyutov, and V. L. Pokrovsky, Phys. Rev. B 66, 014414 (2002).

${ }^{10}$ A. F. Kayali, Phys. Lett. A, 298, 432 (2002).

${ }^{11}$ R. Sasik and T. Hwa, cond-mat/0003462.

12 I. K. Marmorkos, A. Matulis, and F. M. Peeters, Phys. Rev. B 53, 2677 (1996).

${ }^{13}$ Sa-Lin Cheng and H. A. Fertig, Phys. Rev. B 60, 13107 (1999). 
14 J. H. Xu, J. H. Miller, Jr., and C. S. Ting, Phys. Rev. B 51, 424 (1995).

15 J. C. Wei, J. L. Chen, L. Horng, and T. J. Yang, Phys. Rev. B 54, 15429 (1996).

${ }^{16}$ Mark W. Coffey, Phys. Rev. B 52, R9851 (1995).

${ }^{17}$ D. J. Morgan and J. B. Ketterson, Phys. Rev. Lett. 80, 3614 (1998).

${ }^{18}$ M. J. Van Bael, J. Bekaert, K. Temst, L. Van Look, V. V. Moshchalkov, Y. Bruynseraede, G. D. Howells, A. N. Grigorenko, S. J. Bending, and G. Borghs, Phys. Rev. Lett. 86, 155 (2001).

${ }^{19}$ A. S. Mel'nikov, Yu. N. Nozdrin, I. D. Tokman, and P. P. Vysheslavtsev, Phys. Rev. B 58, 11672 (1998).

${ }^{20}$ S. H. Autler, J. Low Temp. Phys. 9, 241 (1972).

${ }^{21}$ M. J. Stephen and J. Bardeen, Phys. Rev. Lett. 14, 112 (1965).

22 B. J. Baelus, F. M. Peeters, and V. A. Schweigert, Phys. Rev. B 61, 9734 (2000).

${ }^{23}$ M. V. Milošević, S. V. Yampolskii, and F. M. Peeters, Physica C 369, 343 (2002); Phys. Rev. B (2002).

24 J. R. Clem, in Proceedings of the 13th Conference on Low Temperature Physics (LT 13), Eds. K. D. Timmerhaus, W. J. O'Sullivan, and E. F. Hammel (Plenum, New York, 1974), Vol. 3, p. 102.

${ }^{25}$ Yu. M. Ivanchenko, A. I. Kozinskaya, and Yu. V. Medvedev, Fiz. Tverd. Tela, 21, 3445 (1979) [Sov. Phys.- Solid State 21, 1989 (1979)].

${ }^{26}$ M. Fusco-Girard and F. Mancini, Physica B\&C 123, 75 (1983).

27 A. Badia, Phys. Rev. B 63, 094502 (2001).

FIG. 1. An oblique view of the systems under investigation with (a) out- and (b) in-plane directed magnetic dipole above a superconducting film interacting with a vortex.

FIG. 2. The interaction energy of the vortex with an out-of-plane magnetic dipole (MD) in units of $F_{0}=\Phi_{0}^{2} /(\pi \lambda)$ as a function of the position of the vortex $\left(\rho_{v}\right)$ : a) for different position of the MD; b) for different thickness of the superconductor; and c) the contour plot of the interaction potential. Inset in (a) illustrates the component of the total MD magnetic field in the presence of the SC, tangential to the SC surface $\left(h_{0}=m_{0} / \lambda^{3}\right)$. Inset in (b) depicts the asymptotic behavior for specific values of the parameters.

FIG. 3. The interaction energy of the vortex with an in-plane magnetic dipole (MD) in units of $F_{0}=\Phi_{0}^{2} /(\pi \lambda)$ as a function of the position of the vortex $\left(\rho_{v}\right)$ : a) for different vertical position of the MD; b) for different thickness of the superconductor; and c) the contour plot of the interaction potential. Open circles denote the energetically favorable position of the vortex.

FIG. 4. The position of the vortex $\left(\rho_{v}^{*}\right)$ as function of the thickness of the superconductor $(d)$ and distance between the in-plane magnetized dipole and the SC surface $(l=a-d / 2) . \rho_{v}^{*}$ is scaled by $l$ or by $\lambda$ (inset of (b)).

FIG. 5. The screening current density in the SC film with $d=0.5 \lambda$ for: a) out-of-plane and b) in-plane magnetized magnetic dipole with $m=m_{0}$ positioned at $a=1.0 \lambda$ above the superconducting film.

FIG. 6. The total interaction energy (in units of $F_{0}=\Phi_{0}^{2} /(\pi \lambda)$ ) between the in-plane magnetic dipole (MD) and the vortex and the antivortex placed at opposite sides of the dipole for different values of the magnetic moment $m$.

FIG. 7. The interaction energy of a vortex-antivortex pair and an in-plane MD, as function of the position of the vortex $\left(\vec{\rho}_{a v}=-\vec{\rho}_{v}\right)$, along the direction of the magnetization of the dipole for: a) different values of the vorticity of the vortex and the antivortex, and b) for single-quantized vortices but different value of the magnetization of the dipole. The open circles denote the local minimum of the interaction energy.

FIG. 8. The position of the vortex-antivortex pair $\left(\rho_{v}^{*}\right)$ as function of the distance between the in-plane magnetized dipole and the SC surface $(l=a-d / 2)$, for different values of the magnetization of the dipole.

FIG. 9. The total interaction energy between an out-of-plane magnetic dipole (MD), the vortex $(+)$ under it and the antivortex (-) at a distance $\rho_{v}$. This illustrates vortex-antivortex pair stabilization by the short range interaction with the MD. 
FIG. 10. The energetically favorable position of the vortex $(+)$ and the antivortex $(-)$ in the case of $N=3$ pairs placed symmetrically around the out-of-plane magnetic dipole (MD). The inset illustrates the configuration.

FIG. 11. The stability phase diagram for $N$ vortex-antivortex pairs around the out-of-plane magnetized dipole, as function of the distance between the dipole and the SC surface $(l)$ and the magnetic moment $m$. Solid lines denote the critical value of the moment needed for the stability of such vortex-antivortex configurations.

FIG. 12. The free energy difference between the states with $N$ vortex-antivortex pairs around the dipole and the Meissner state, for a thin SC film with $\lambda / \xi=10$ and the out-of-plane dipole positioned at $l / \lambda=1.0$. Inset shows the total interaction energy in the corresponding $N$-states. 


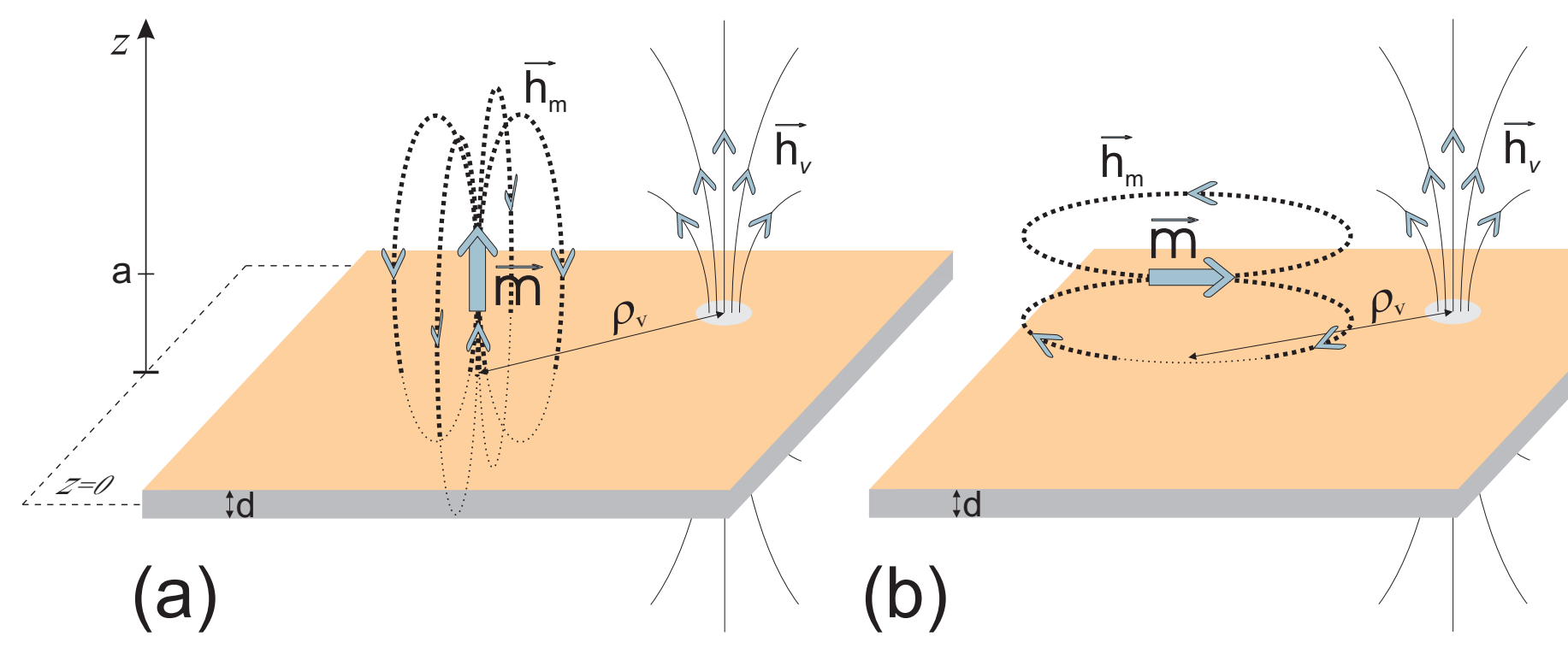

Fig. 1 Milosevic et al. 


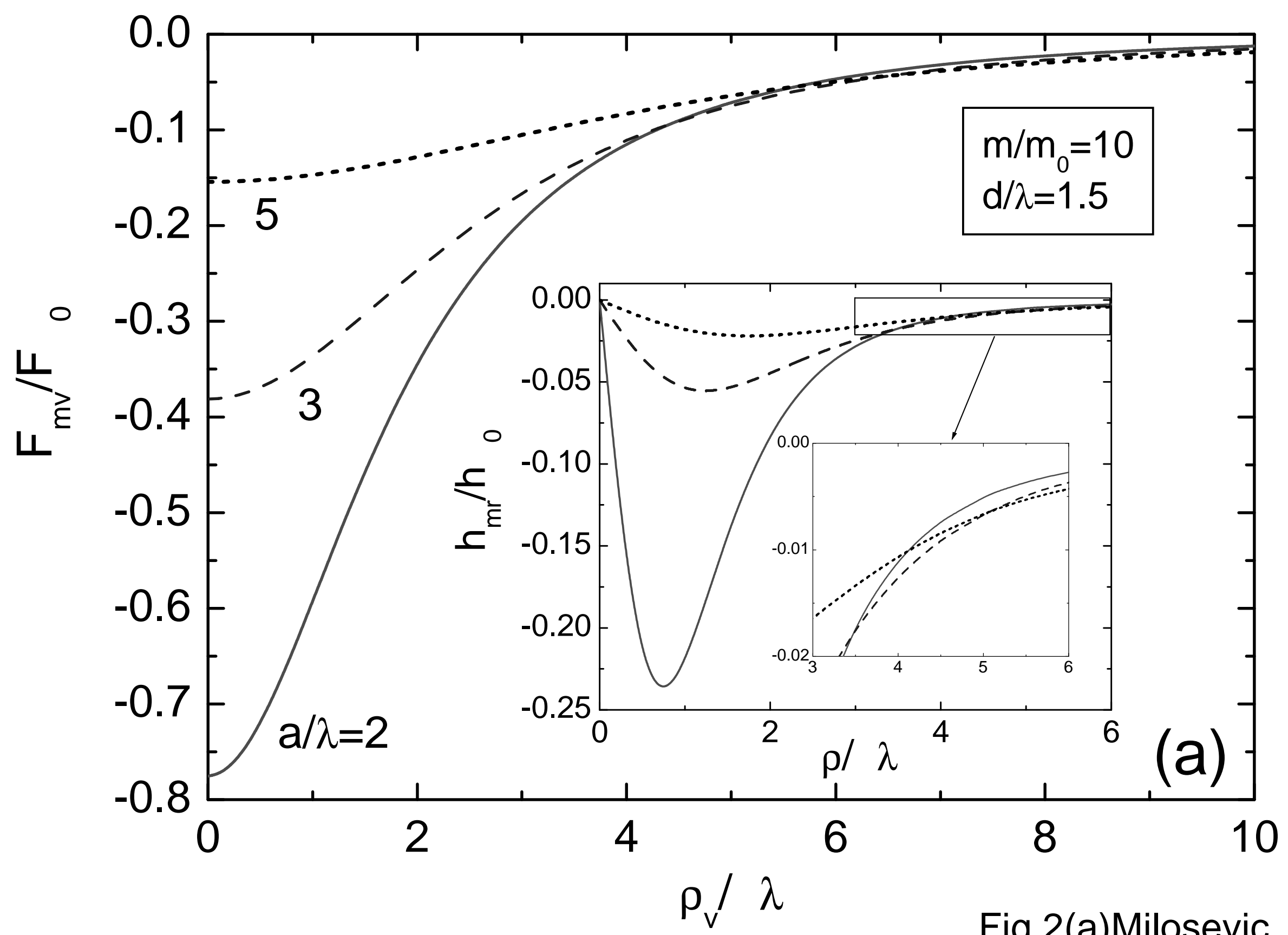

Fig. 2(a) Milosevic et al. 


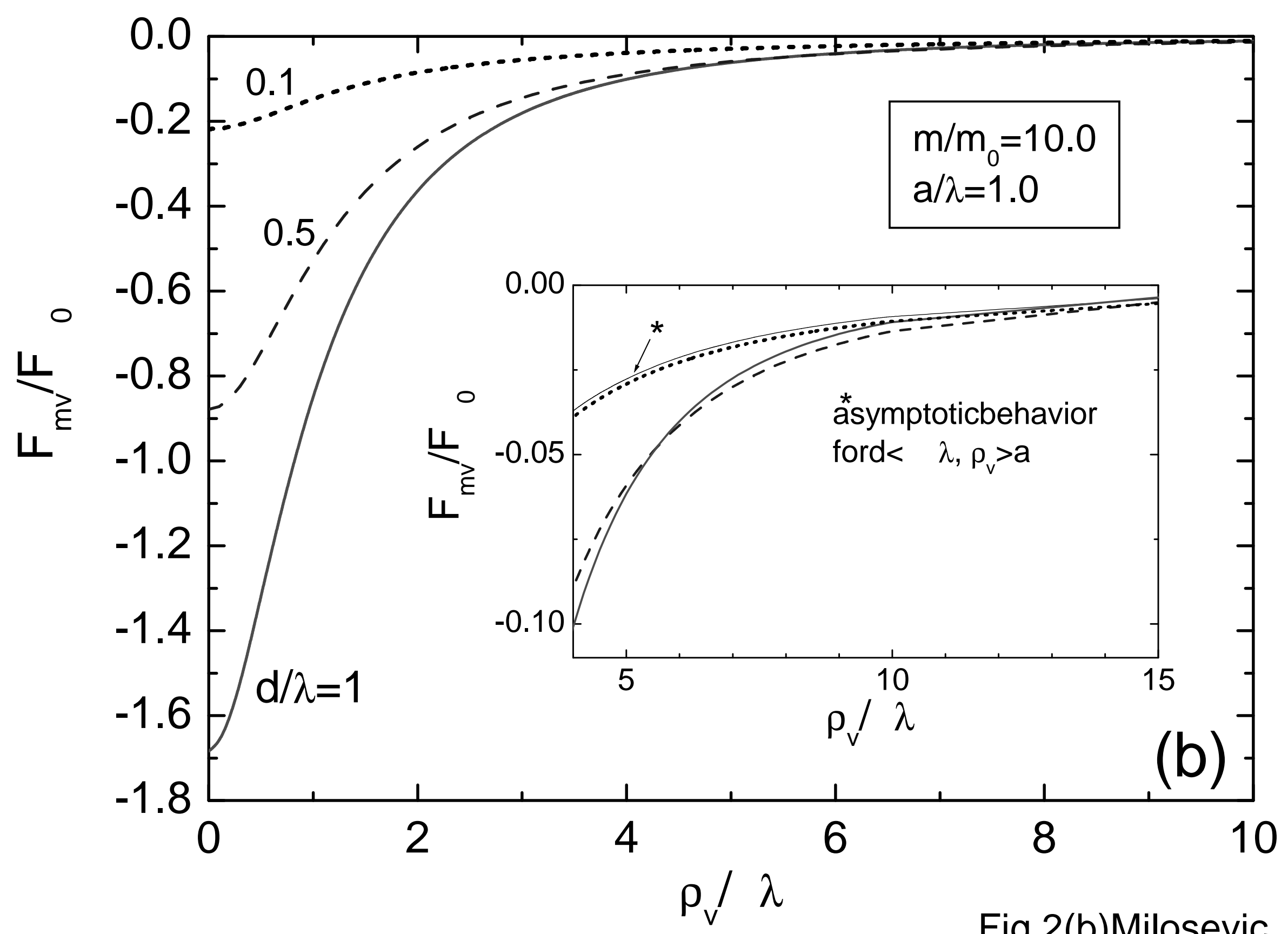

Fig. 2(b) Milosevic et al. 


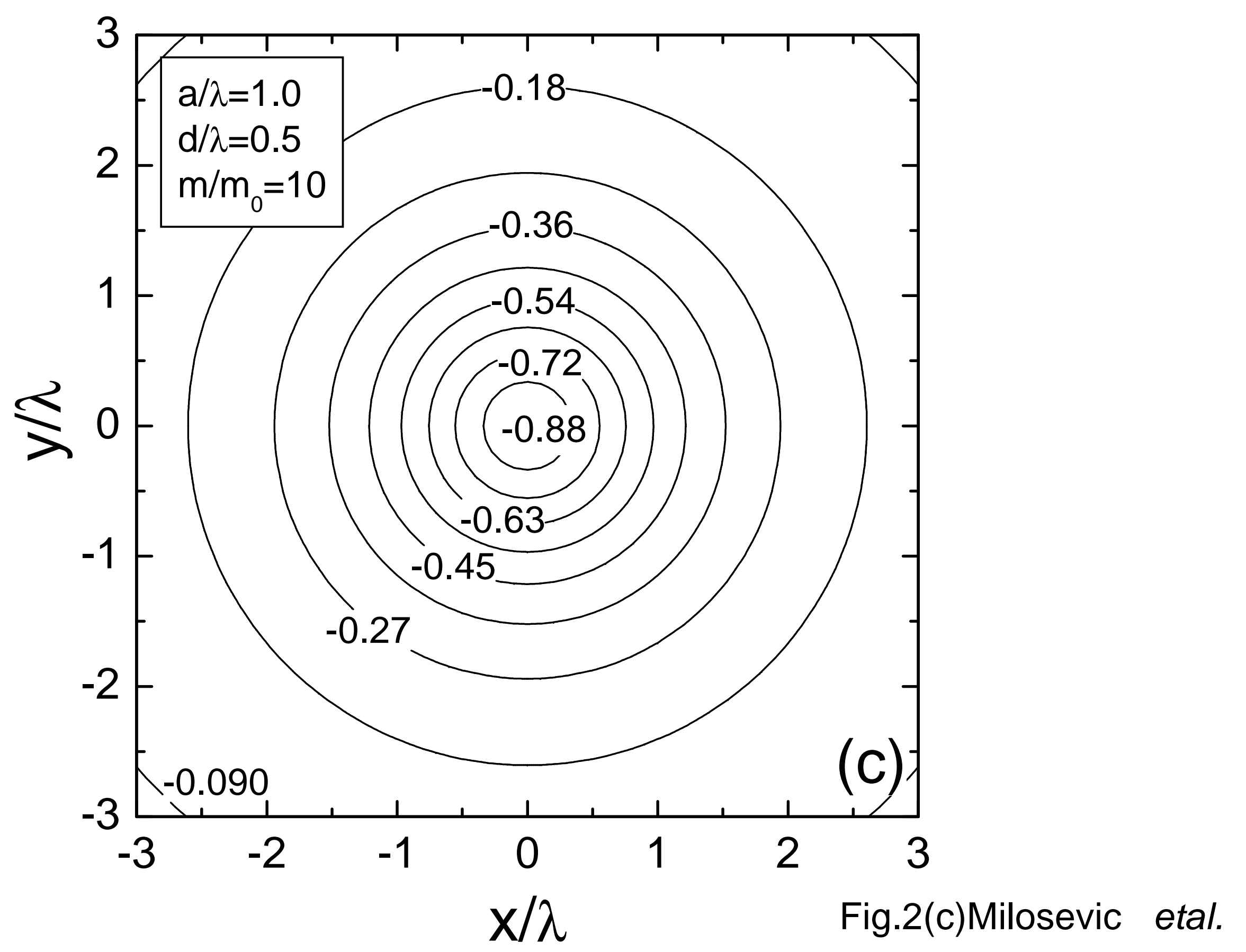




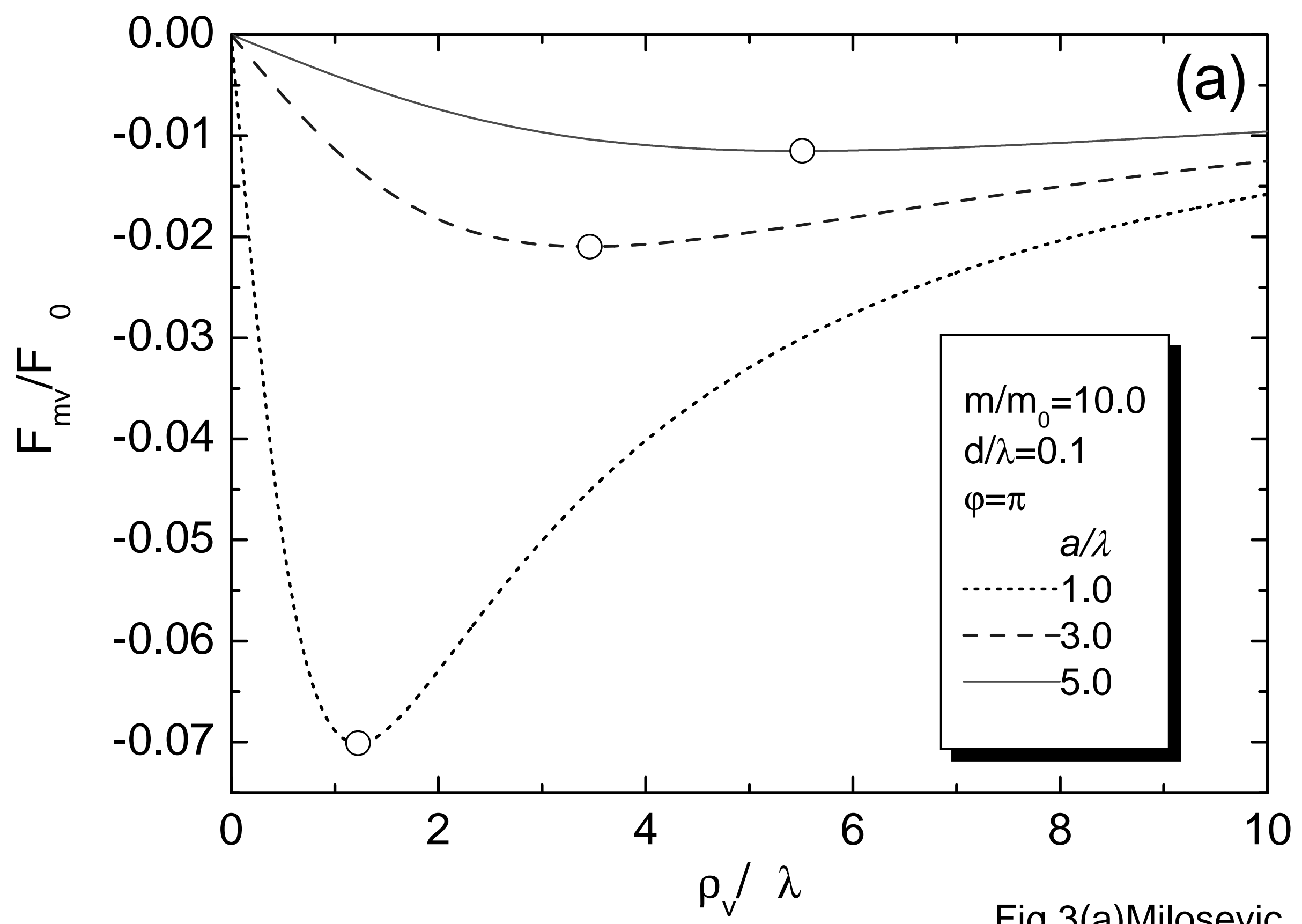

Fig. 3(a) Milosevic et al. 


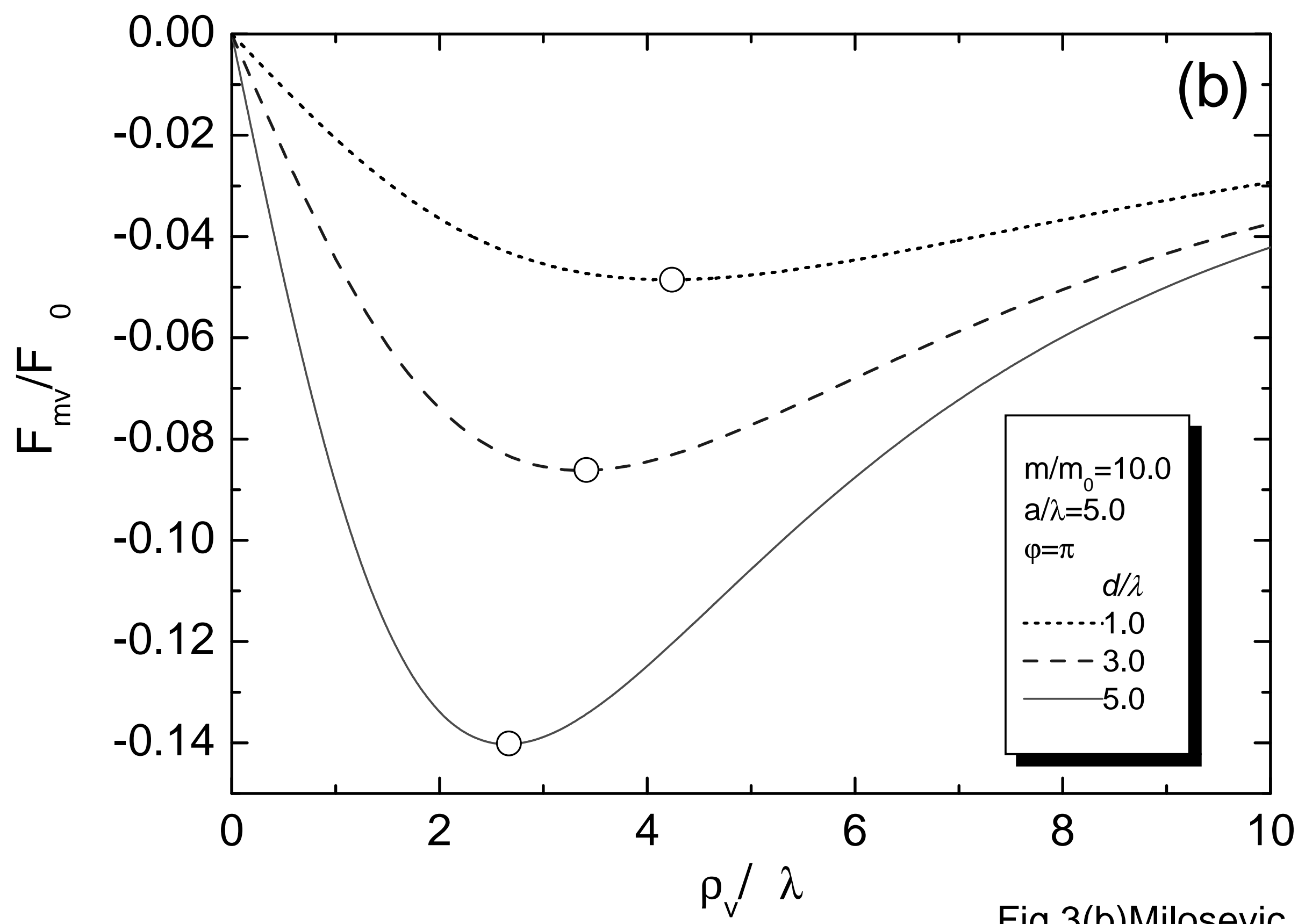

Fig. 3(b) Milosevic et al. 


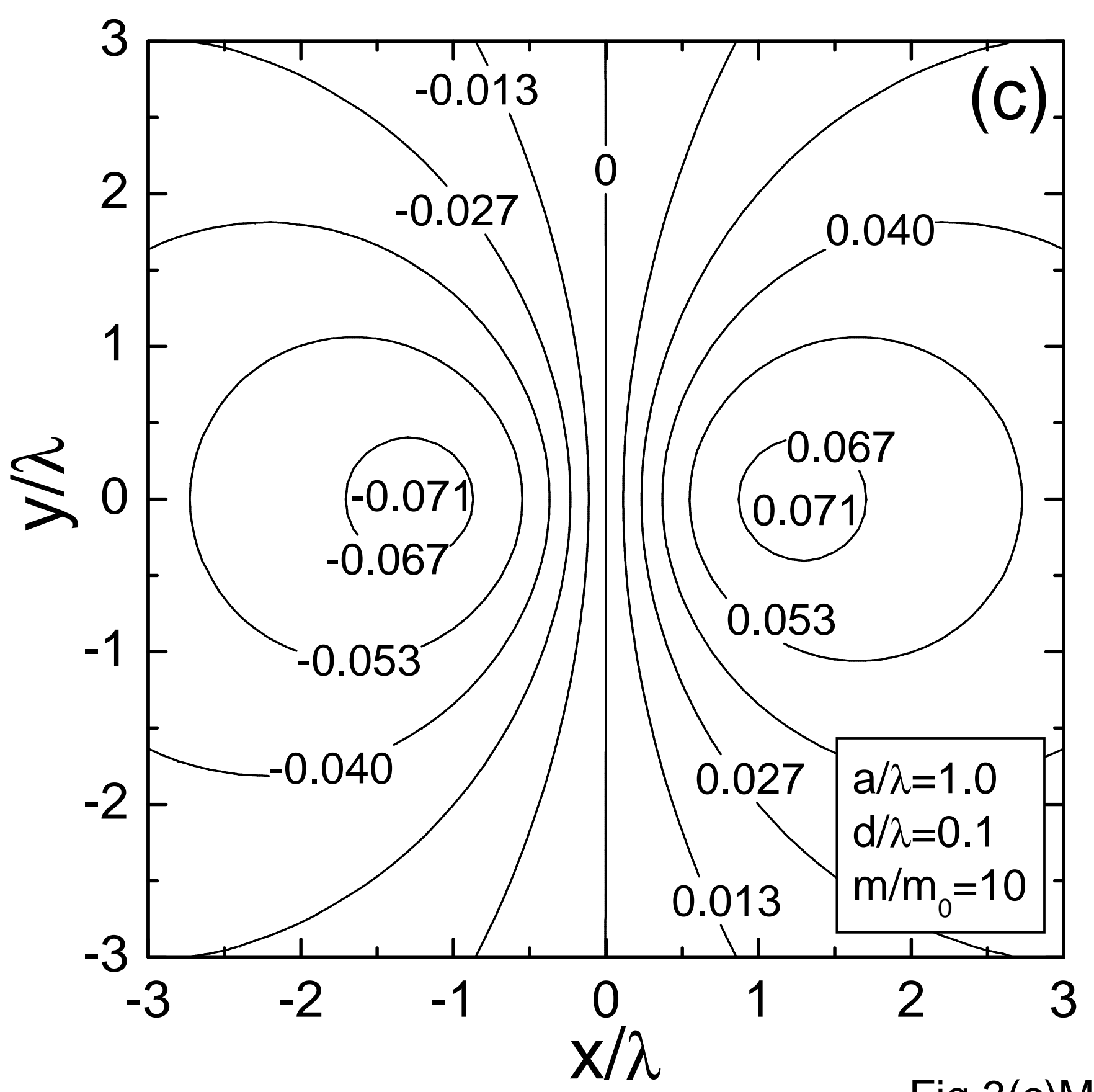

Fig. 3(c) Milosevic et al. 


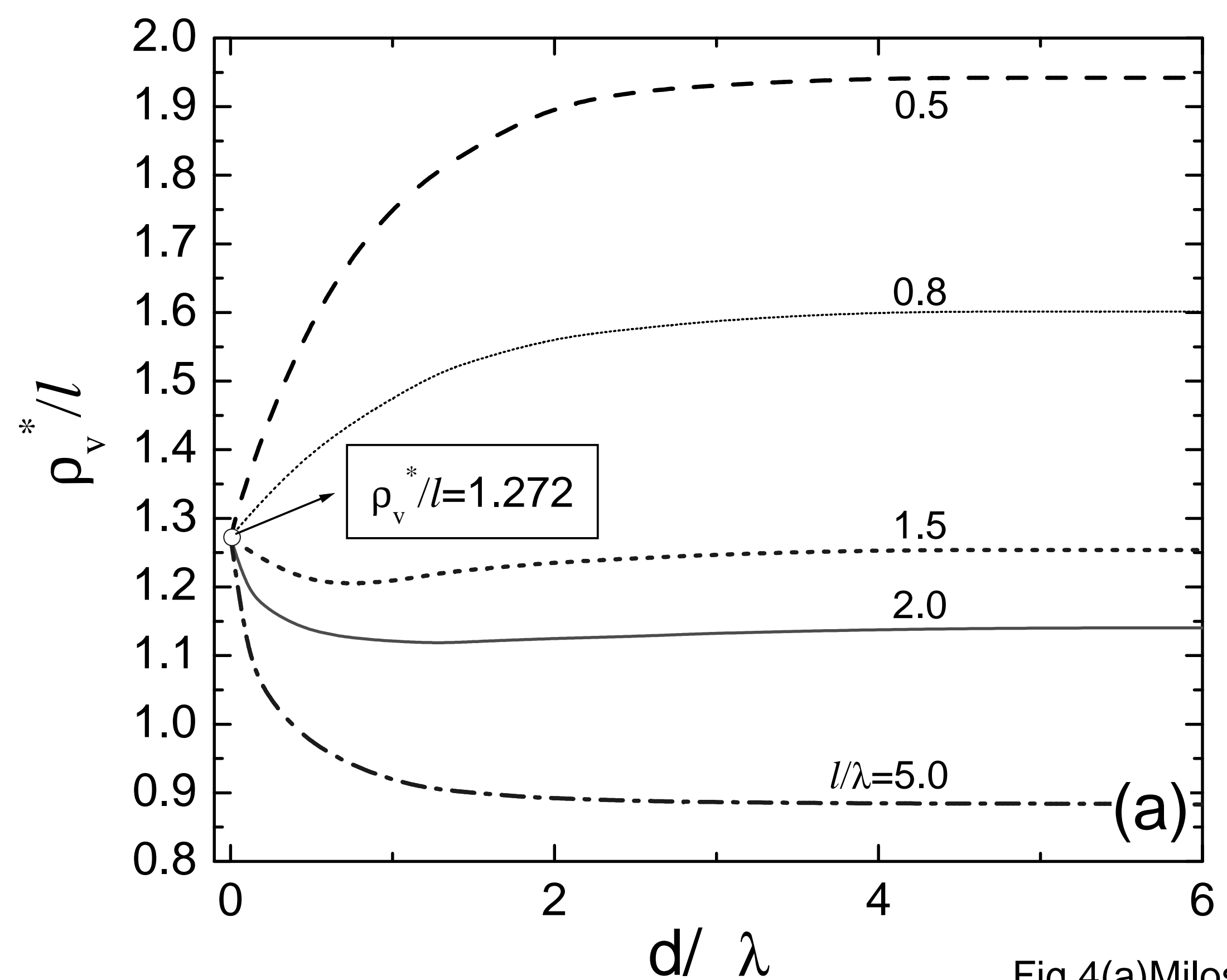

Fig. 4(a) Milosevic et al. 


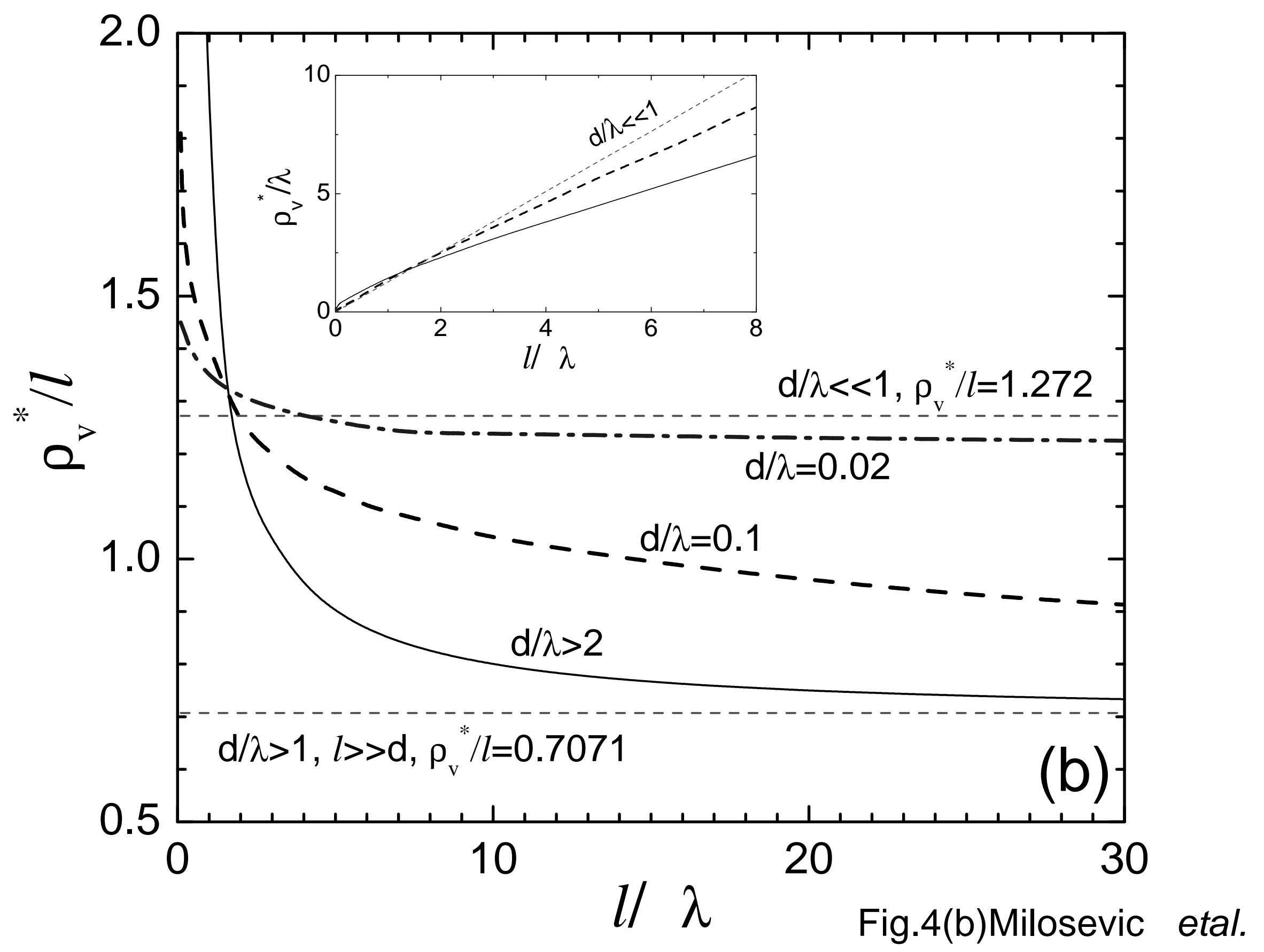



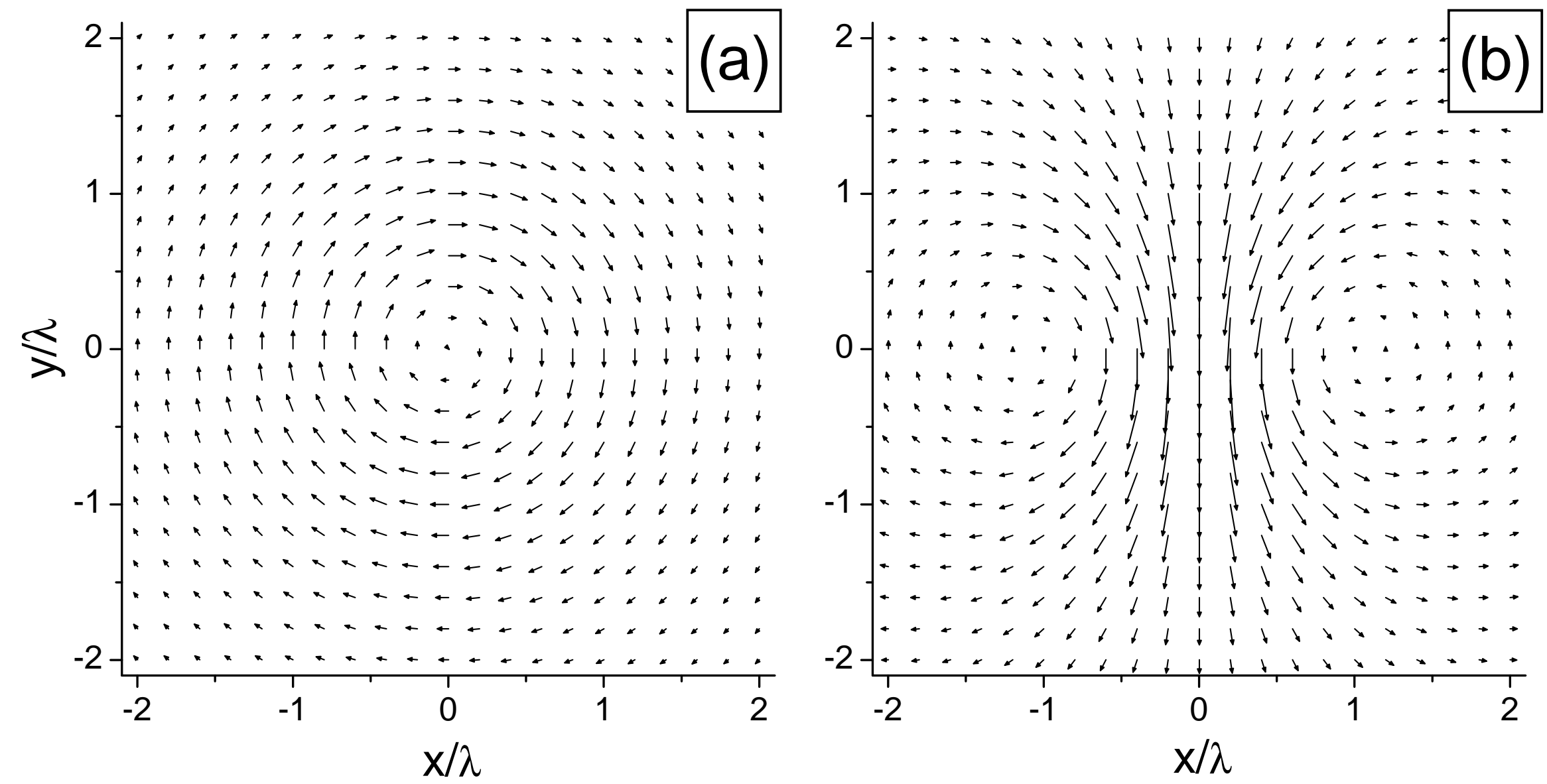

Fig. 5 Milosevic et al. 


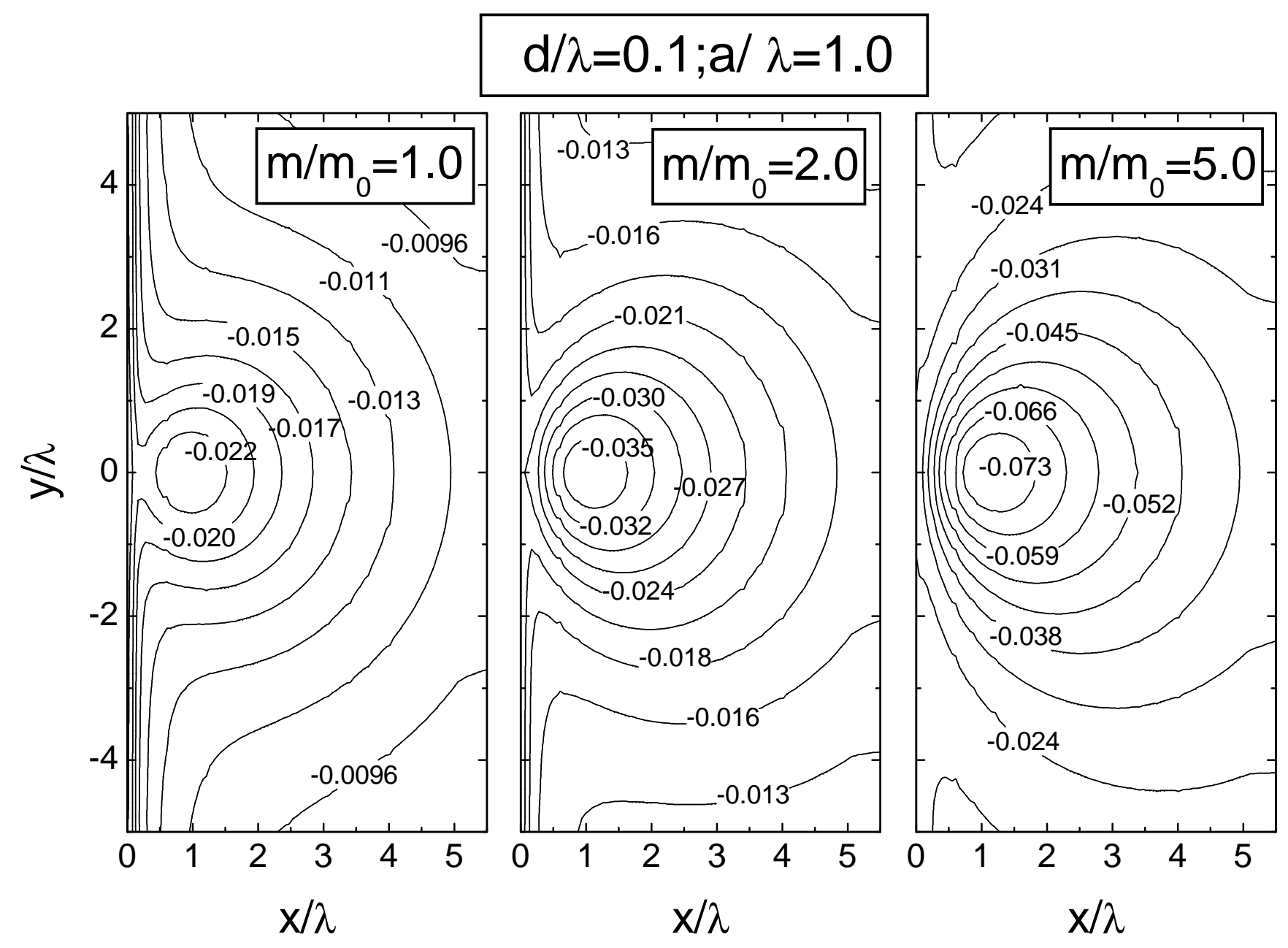

Fig. 6 Milosevic et al. 


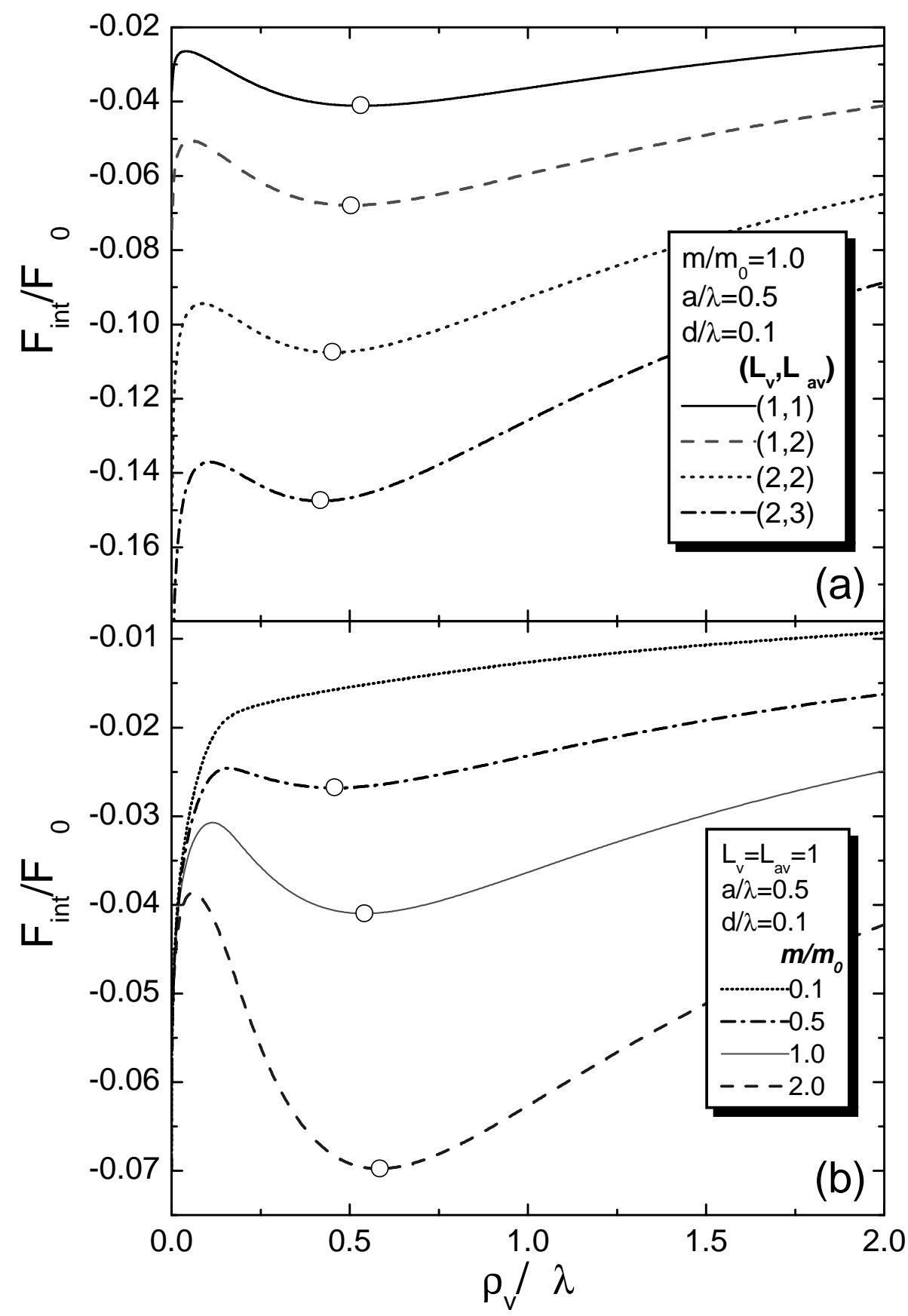

Fig. 7 Milosevic et al. 


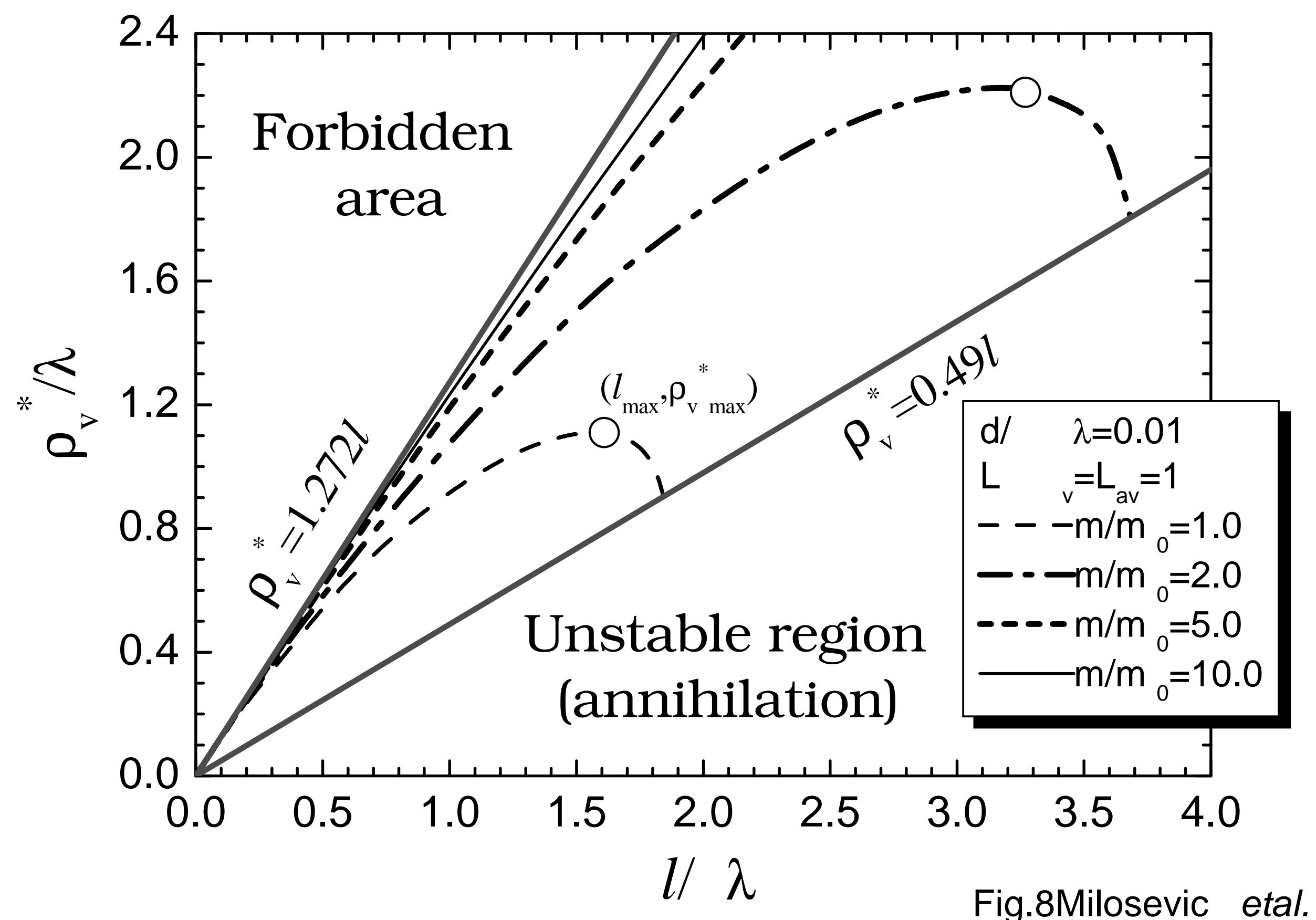

Fig. 8 Milosevic et al. 


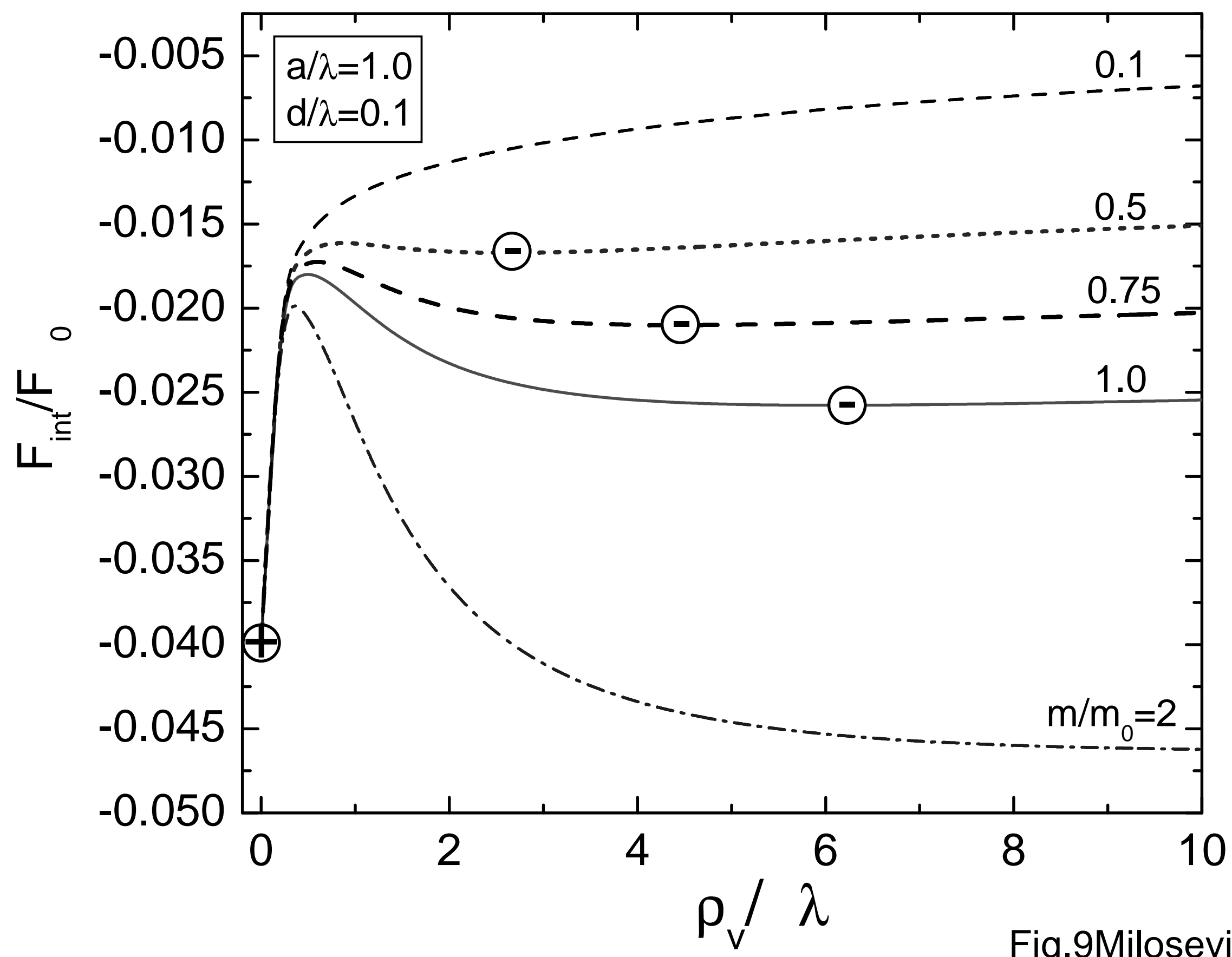

Fig. 9 Milosevic et al. 


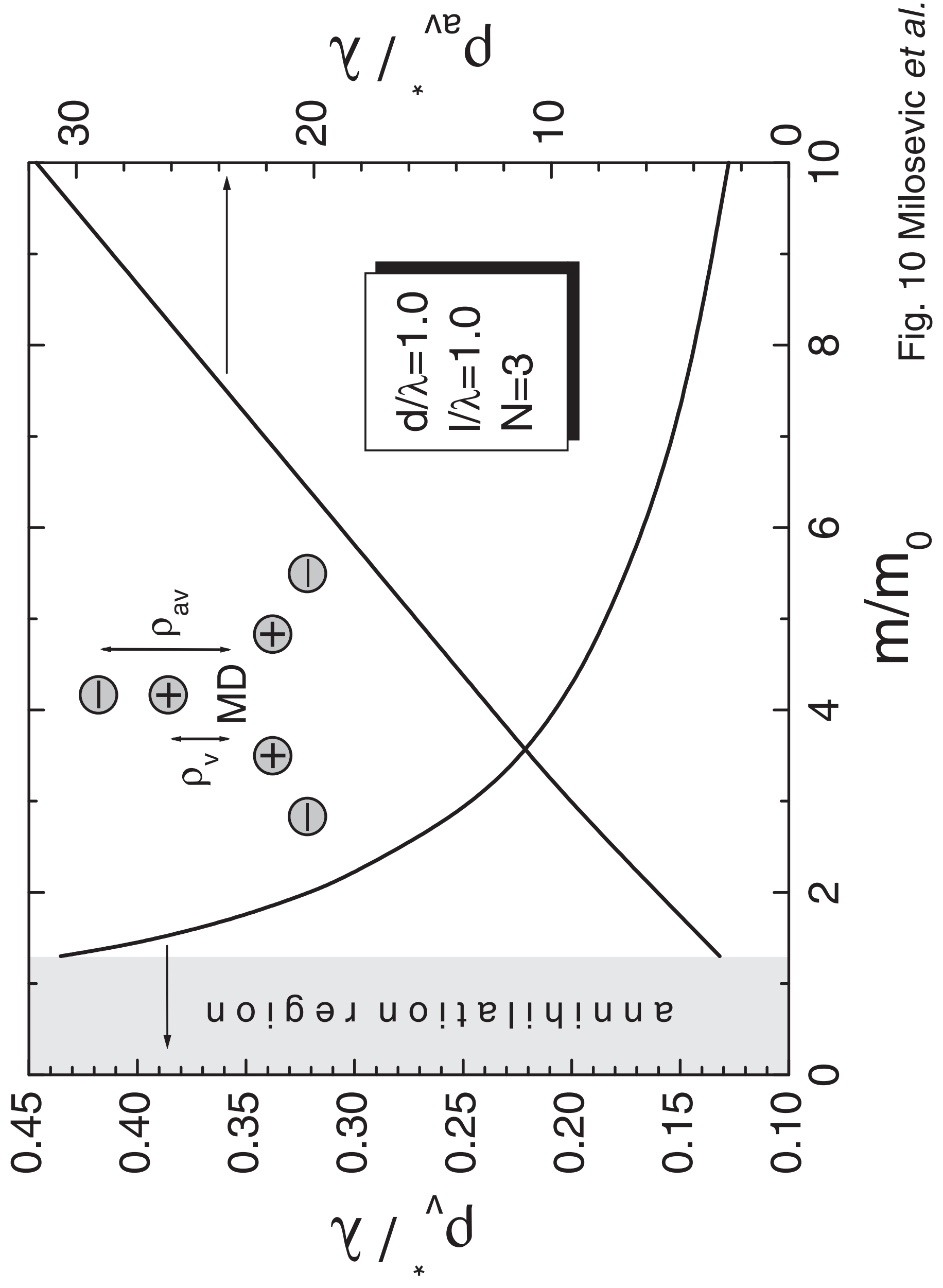




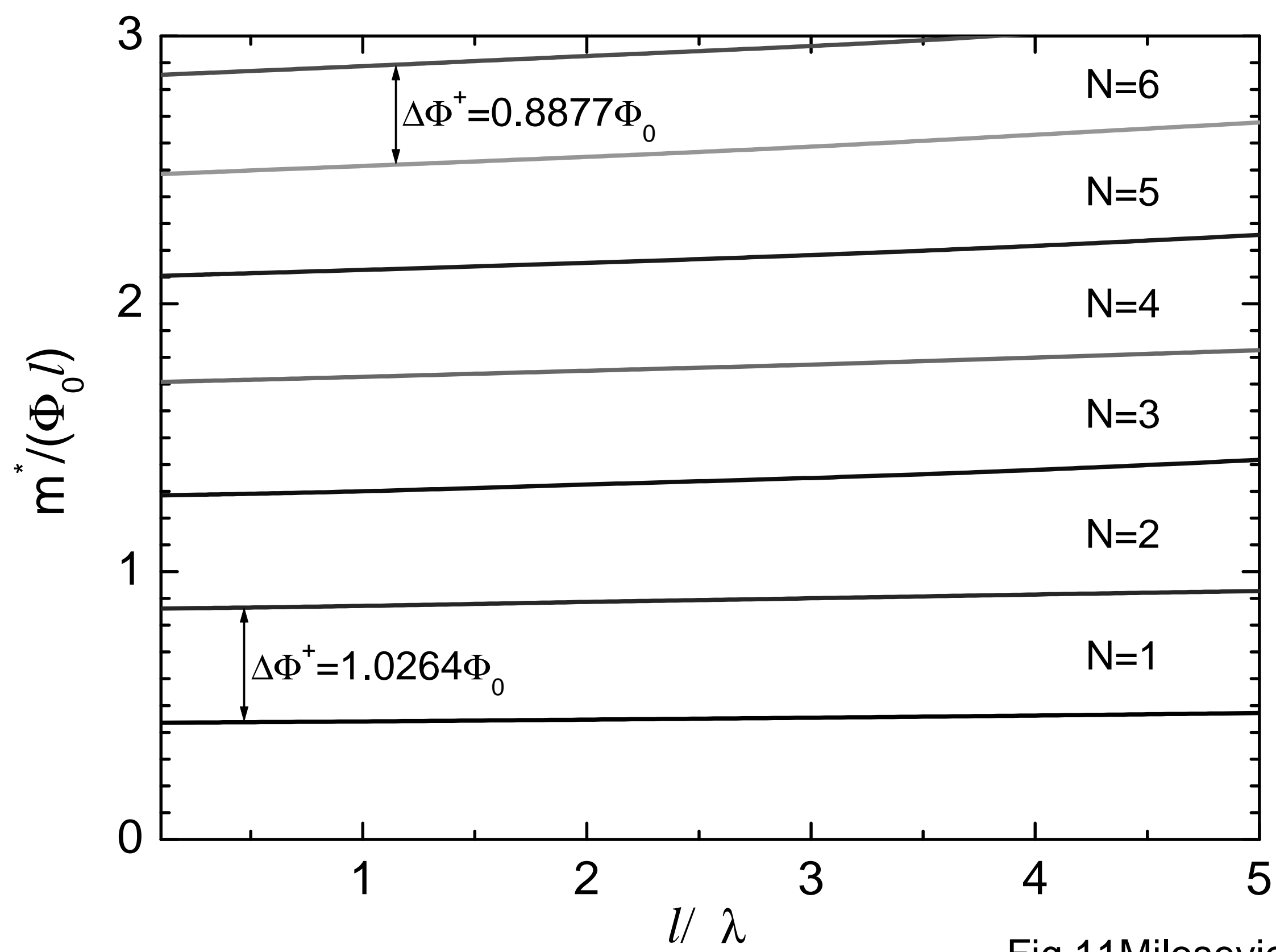

Fig. 11 Milosevic et al. 


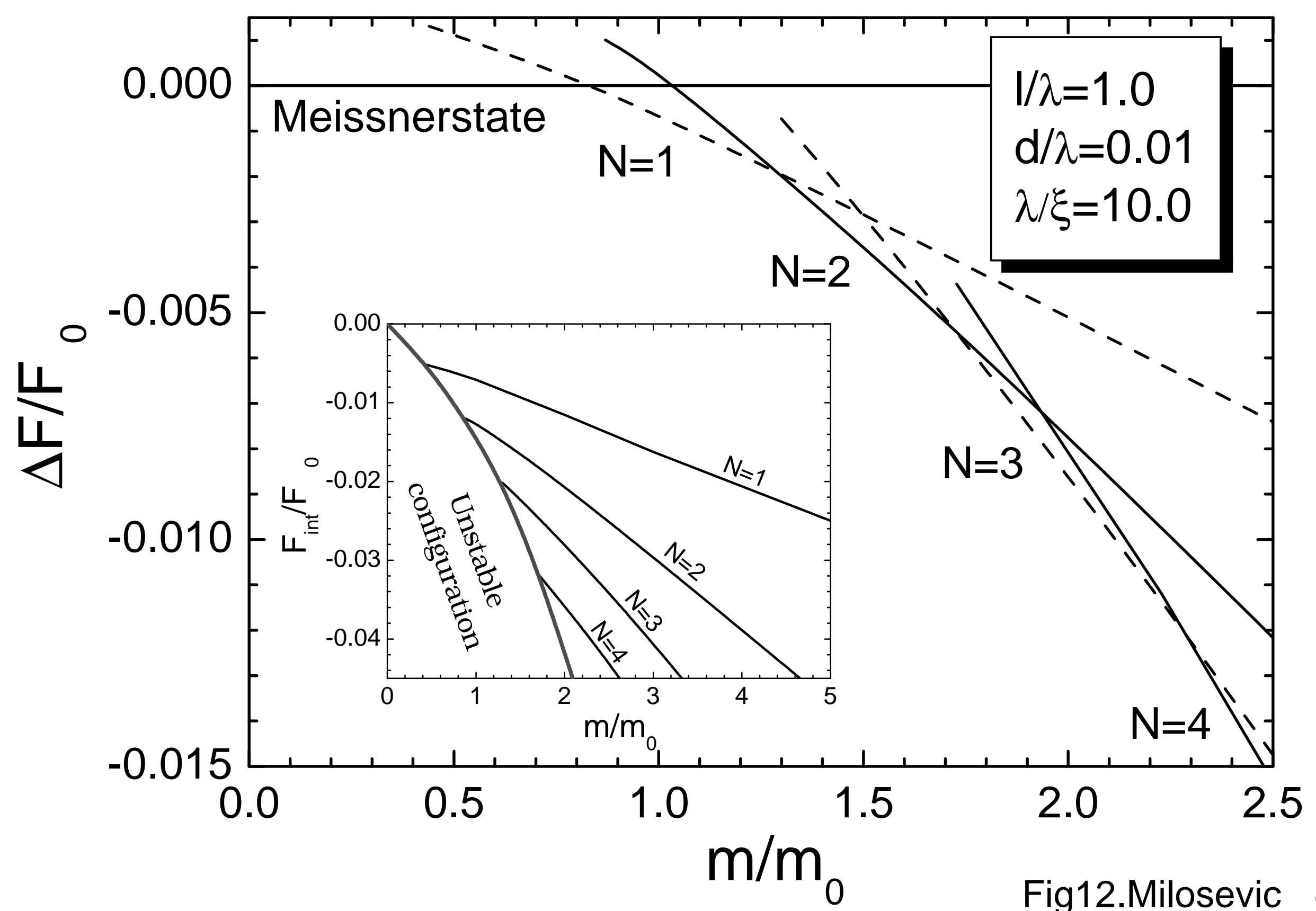

Fig 12. Milosevic et al. 